\title{
A vesicle microrheometer for high-throughput viscosity measurements of lipid and polymer membranes
}

\author{
Hammad A. Faizi ${ }^{1}$, Rumiana Dimova ${ }^{2}$ and Petia M. Vlahovska ${ }^{3}$ \\ 1 Department of Mechanical Engineering, Northwestern University, Evanston, IL 60208, USA \\ ${ }^{2}$ Department of Theory and Biosystems, Max Planck Institute of \\ Colloids and Interfaces, Science Park Golm, 14424 Potsdam, Germany \\ ${ }^{3}$ Department of Engineering Sciences and Applied Mathematics, \\ Northwestern University, 60208, USA, email: petia.vlahovska@northwestern.edu
}

(Dated: September 7, 2021)

\begin{abstract}
Viscosity is a key property of cell membranes that controls mobility of embedded proteins and membrane remodeling. Measuring it is challenging because existing approaches involve complex experimental designs and/or models, and the applicability of some is limited to specific systems and membrane compositions. As a result there is scarcity of data and the reported values for membrane viscosity vary by orders of magnitude for the same system. Here, we show how viscosity of bilayer membranes can be obtained from the transient deformation of giant unilamellar vesicles. The approach enables a non-invasive, probe-independent and high-throughput measurement of the viscosity of bilayers made of lipids or polymers with a wide range of compositions and phase state. Pure lipid and single-phase mixed bilayers are found to behave as Newtonian fluids with strain-rate independent viscosity, while phase-separated and diblock-copolymers systems exhibit shear-thinning in the explored range of strain rates 1-2000 $\mathrm{s}^{-1}$. The results also reveal that electrically polarized bilayers can be significantly more viscous than charge-neutral bilayers. These findings suggest that biomembrane viscosity is a dynamic property that can be actively modulated not only by composition but also by membrane polarization, e.g., as in action potentials.
\end{abstract}

Cells and cellular organelles are enveloped by membranes, whose main structural component is a lipid bilayer [1. The lipid bilayer endows membranes with fluidity that is essential for functions that depend on biomolecules mobility, e.g., signaling 244 . Fluidity is modulated by membrane composition and this homeoviscous adaptation is crucial for the survival of organisms that can not regulate their body temperature like bacteria [5, 6]. Viscosity is the common measure for fluidity, yet for membranes this property has been challenging to assess. Data for viscosity of lipid membranes are limited and reported values vary significantly, sometimes by orders of magnitude for the same system (SI Table 1). For example, reported values for the surface shear viscosity of membranes made of a typical lipid such as dioleoylphosphatidylcholine (DOPC) span two orders of magnitude: $(0.197 \pm 0.0069) \times 10^{-9}$ Pa.s.m [7, $(1.9 \pm 11) \times 10^{-9}$ Pa.s.m 8, $(16.72 \pm 1.09) \times 10^{-9}$ Pa.s.m 9]. For a similarly structured lipid, palmitoylphosphatidylcholine (POPC), the surface viscosity measured by shear rheology of Langmuir monolayers is $3 \times 10^{-4}$ Pa.s.m [10]. Experimental methods that utilize free-standing bilayer membranes, e.g, vesicles or black lipid membranes, rely on estimates from the rate of tether formation [11, diffusion coefficients of domains 12 14 or membraneanchored nanoparticles [15, 16, domain shape fluctuations [17, domains motion on vesicles induced by applied flow [8, 18, bilayer thickness fluctuations or lipid dynamics measured with neutron spin echo spectroscopy [1921, fluorescence quantum yield or lifetime of viscositysensitive fluorescent dyes 22, 23, and the forced motion of colloidal particles in the membrane 24 26]. In silico approaches, using molecular dynamics simulations, have also been developed to determine membrane viscosity [7, 27, 28. Despite these advances, the systematic study of membrane viscosity has been hindered by various limitations of the proposed methodologies. For example, domain-based methods [8, 12, 13, 17, 18, are limited to phase-separated membranes and the measured viscosity reflects the continuous phase, not the membrane as a two-phase fluid. Bilayer thickness fluctuations [19] depend on both shear and dilational monolayer viscosities. In particle-based methods [15, 16, 24 26], the probe perturbs the membrane and the data interpretation requires complicated analysis that discerns the contributions to the particle mobility from the flow in the membrane and the surrounding fluids 29 31. Furthermore, since membrane surface viscosity is a macroscopic quantity, defined on scales where the bilayer can be modeled as a twodimensional incompressible fluid, methods utilizing measurements at the micro- or nano- scale and/or based on molecular probes may not report the effective continuum viscosity but a quantity, often called "microviscosity", which is local and depends on the immediate environment [21. These complexities are likely the source of the huge variability in reported values of viscosity for lipid bilayer membranes, making it challenging to compare data obtained by different methods.

Here, we show that the deformation of giant unilamellar vesicles (GUVs) can be employed to obtain the surface "macroviscosity", i.e., the shear viscosity of the membrane treated as a two-dimensional incompressible fluid. Upon application of an extensional stress, e.g., generated by an uniform electric field [32 35], extensional flow [36 38, or an optical stretcher [39, 40] a quasi-spherical vesicle deforms into a prolate ellipsoid. The aspect ratio, 
A)

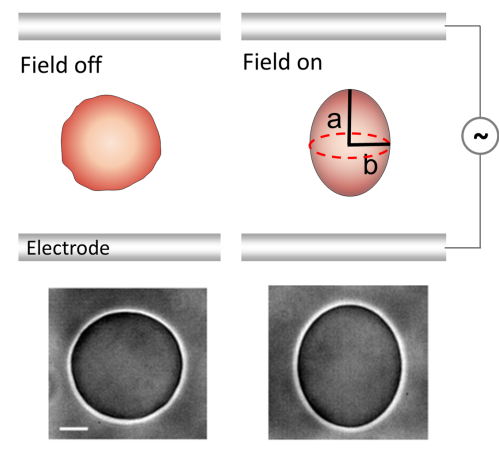

B)

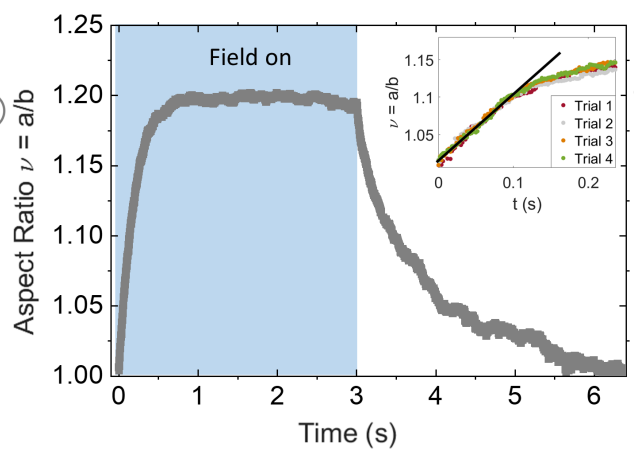

C)

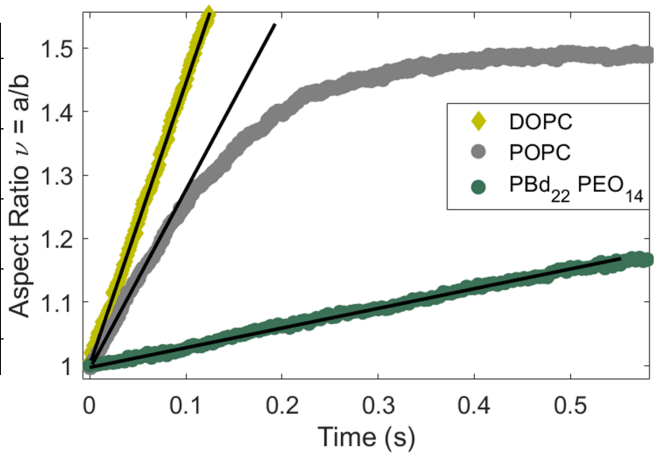

FIG. 1. Electrodeformation method to measure membrane viscosity. (A) A uniform electric field deforms a GUV into a prolate ellipsoid by pulling out area stored in suboptical thermally-excited membrane undulations. Snapshots of the vesicle during the experiment. Imaging with phase contrast microscopy. Scale bar: $15 \mu \mathrm{m}$. (B) Prolate deformation of a POPC GUV in an electric field with amplitude $E_{0}=10$ $\mathrm{kV} / \mathrm{m}$ and frequency of $1 \mathrm{kHz}$. Time zero in all graphs corresponds to turning the field on. The inset shows that repeated deformation does not alter the initial slope of the deformation curve. C) Vesicles made of lipids (DOPC, POPC) and the diblock-copolymer $\mathrm{PBD}_{22} \mathrm{PEO}_{14}$ deform at a different rate indicating different membrane viscosity. The field strength and frequency are $8 \mathrm{kV} / \mathrm{m} \mathrm{and} 1 \mathrm{kHz}$. The solid lines correspond to the theoretical fit with Eq. 2

$\nu=a / b$ (see sketch in Fig. 1A), increases and reaches a steady state. When the stress is removed, the vesicle relaxes back to its equilibrium spherical shape, see Fig. 11B. The reproducibility of the results was tested by repeated measurements with the same vesicle, see Fig. $1 \mathrm{~B}$ inset, showing identical slope of the aspect ratio curves for small deformations.

Even though the applied stress is extensional and vesicle deformation is axisymmetric, material transported on the vesicle surface undergoes shear because the membrane is area-incompressible [41-45]. The rate at which the vesicle elongates while the field is on, and relaxes back to its equilibrium shape after the field is turned off, is related to the membrane shear viscosity. For small deformations, $\nu \lesssim 1.3$, the evolution of the aspect ratio is described by [34, 45,47] (see Appendix Section 2 for a summary of the theory)

$$
\dot{\nu}=\frac{1}{\eta\left(55+16 \chi_{m}\right)}\left(p-\frac{24 \sigma}{R}(\nu-1)\right)
$$

where $\chi_{m}=\eta_{m} / \eta R$ is the dimensionless surface viscosity $\eta_{m}, \eta$ is the viscosity the solution inside and outside the vesicle (assumed to be the same), $\sigma$ is the membrane tension, and $R$ is the vesicle radius. In an applied extensional flow with strain rate $\dot{\gamma}, p=180 \eta \dot{\gamma}$. In the case of a charge-neutral vesicle in a uniform DC electric field with amplitude $E_{0}, p=27 \varepsilon E_{0}^{2} / 4$, and in an AC field with frequency $\omega, p(\omega)$ is given in Appendix Section 2. Thus from the vesicle dynamics in response to an extensional flow or a uniform electric field it is straight-forward to obtain the membrane viscosity. Note that the dynamics does not involve dilational viscosity because vesicle deformation and the accompanying increase in apparent area comes from ironing of suboptical thermally-excited membrane undulations, while the area per lipid remains the same.

\section{RESULTS}

We illustrate the implementation of the approach on the example of a quasi-spherical vesicle subjected to a uniform AC electric field. The electric field is advantageous over extensional flow or radiation pressure in optical stretchers because of (i) the simplicity of the experimental set up, and (ii) the ability to create potential difference across the bilayer emulating transmembrane potentials in living cells. The use of an AC field is preferable because (i) a DC field could cause Joule heating and electroosmotic flows, whose effect on vesicle deformation and stability is difficult to account for [48, and (ii) the transmembrane potential can be modulated by changing the electric field frequency [49, 50]. We applied the method to fluid membranes composed of the phosphatidylcholine lipids palmitoyloleoyl (POPC), dioleoyl (DOPC), oleoylmyristoyl (OMPC), stearoyloleoyl (SOPC), and dipalmitoyl (DPPC), cholesterol (chol) and diblock copolymers, $\mathrm{PBd}_{x}-b-\mathrm{PEO}_{y}$ with varying hydrophobic molecular weight, $\mathrm{M}_{h}$, from $0.7 \mathrm{kDa}$ to 6.8 $\mathrm{kDa}$.

\section{Vesicle transient deformation yields membrane viscosity.}

Figure 1 summarizes the experiment. The elongation curves of a GUV initially show linear increase (Figure $1 \mathrm{C}$ and Appendix Figure S3). The linear slope is predicted by Eq. 1 if the second term, which describes the action of the tension opposing the deformation, is neglected

$$
\nu=1+\frac{t}{t_{\mathrm{d}}}\left(\frac{p \omega)}{\left(55+16 \chi_{m}\right)}\right)
$$



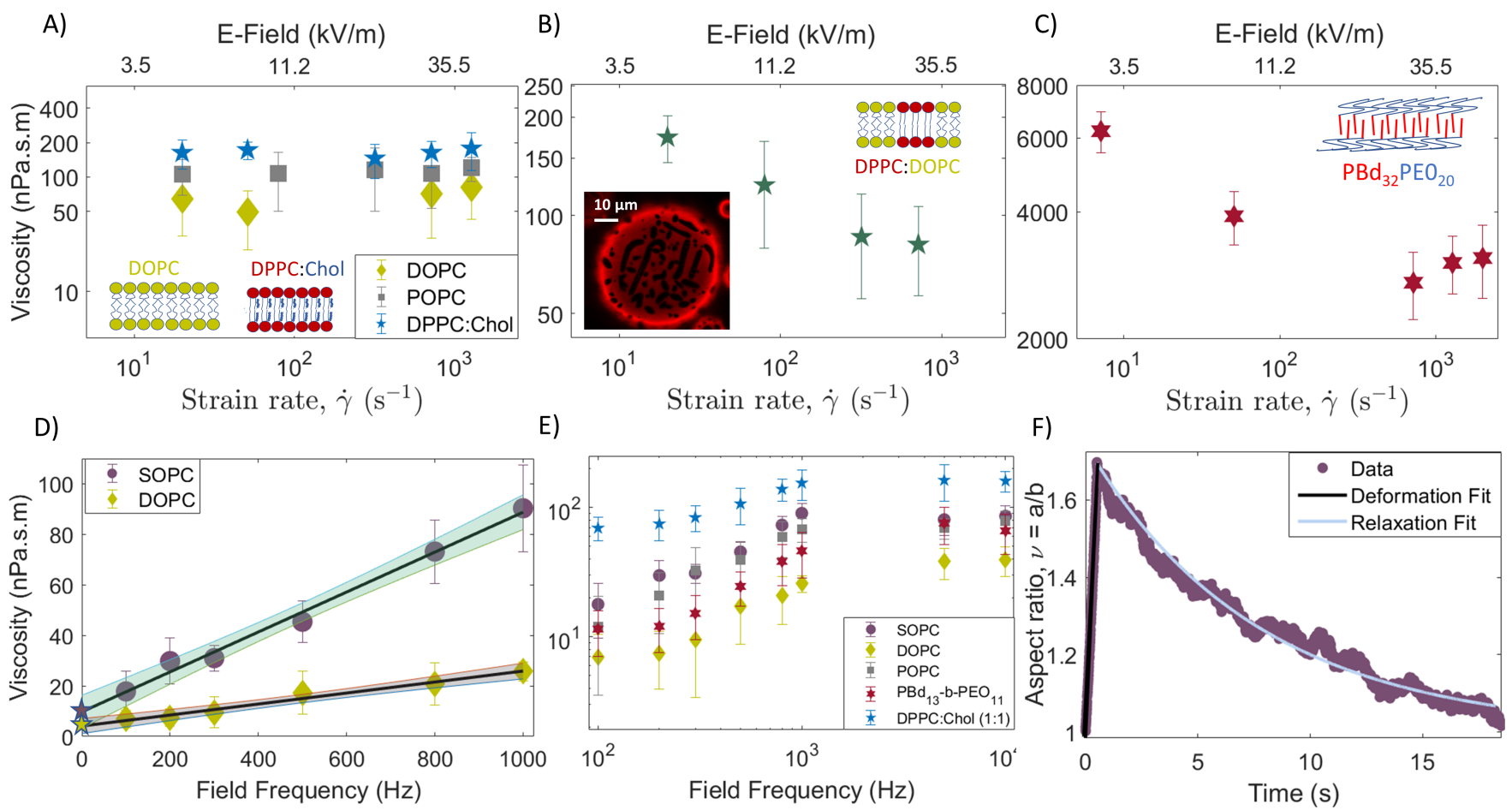

FIG. 2. Viscosity dependence on electric field strength, or equivalently strain rate $\dot{\gamma}=\varepsilon E_{0}^{2} / \eta$, and frequency. A) Single-component (DOPC or POPC) and single-phase multicomponent bilayers (DPPC:Chol 1:1) behave as Newtonian fluids. B) Viscosity of the phaseseparated multicomponent (DPPC:DOPC 1:1) decreases with the effective strain rate. The inset shows an intricate network of finger-like domains indicative of fluid (red) and gel (dark) phase coexistence. C) Diblock-copolymer bilayers also display shear thinning viscosity. All measurements in panels A-C, were done at field frequency $1 \mathrm{kHz}$. D) Viscosity measured at different frequencies at a fixed strain rate $\dot{\gamma}=50 \mathrm{~s}^{-1}$, corresponding to field strength $8 \mathrm{kV} / \mathrm{m}$, increases with frequency. Extrapolating to zero frequency (the DC limit) yields the shear surface viscosity. The symbols on the vertical-axis-intercept refer to the extrapolated values of $4.11 \pm 2.63 \mathrm{nPa} . \mathrm{s} . \mathrm{m}$ and $9.73 \pm 5.80$ nPa.s.m for DOPC and SOPC, respectively. The R-squared value of the linear fit is 0.98 and 0.97 for the SOPC and DOPC, respectively. The shaded band represents a $95 \%$ confidence interval. E) The viscosity reaches as plateau at frequencies above few kHz. This behavior is common to all studied compositions. F) Shape relaxation after the field is turned off yields the similar value for the viscosity as the zero-frequency limit obtained from the frequency sweep. Data for SOPC, $E_{0}=8 \mathrm{kV} / \mathrm{m}$ and $\omega=1 \mathrm{kHz}$. Tension obtained from flickering spectroscopy is $5.7 \pm 2.6 \times 10^{-9} \mathrm{~N} / \mathrm{m}$. Viscosity obtained from initial deformation and relaxation is $164 \mathrm{nPa} . \mathrm{s} . \mathrm{m}$ and $21.1 \pm 29.4 \mathrm{nPa}$.s.m, respectively.

where $1 / t_{\mathrm{d}}=\varepsilon E_{0}^{2} / \eta$ is the characteristic rate-of-strain imposed by the electric field. Thus, the slope of the aspect ratio plotted as a function of the rescaled time $t / t_{\mathrm{d}}$, at the same field frequency, depends solely on the membrane viscosity. Vesicles made of different lipids or diblock-copolymers show different initial slopes, see Fig. ${ }_{1 \mathrm{C}} \mathrm{C}$, indicating different membrane viscosities. The linear response is observable only if the restoring force of the membrane tension is negligible compared to the deforming electric stress, i.e., $\sigma / R \varepsilon E_{0}^{2} \ll 1$, which is indeed the case for typical values of the applied electric field $E_{0}=5$ $\mathrm{kV} / \mathrm{m}$, equilibrium membrane tension $\sigma=10^{-8} \mathrm{~N} / \mathrm{m}$, and vesicle radius $R=10 \mu \mathrm{m}$. The time up to which the linear approximation is reasonable (see Appendix Section $2 \mathrm{~B})$ is estimated to be $t_{c} / t_{\mathrm{d}} \sim \varepsilon E_{0}^{2} R\left(55+16 \chi_{m}\right) /(12 \sigma)$. Using times up to $0.1 t_{c} / t_{\mathrm{d}}$ (or equivalently $\nu$ up to 1.1 ) minimizes the error in the linear fit.

\section{Viscosity shows dependence on field amplitude and frequency.}

The stress, generated by the electric field, shears the membrane with a characteristic rate $\dot{\gamma}=1 / t_{\mathrm{d}}=\varepsilon E_{0}^{2} / \eta$. Modulating the field amplitude thus enables us to vary the shear rate in a wide range and to probe if bilayers behave as Newtonian fluids: with shear-rate independent viscosity. Increasing $E_{0}$ from 1 to $50 \mathrm{kV} / \mathrm{m}$ at a given frequency increases the effective shear rate from 1 $s^{-1}$ to $2000 s^{-1}$. We find that bilayers made of only one lipid or a homogeneous mixture, either in the liquid disordered (e.g., DOPC or POPC) or liquid ordered (e.g., DPPC:Chol 1:1) state, exhibit rate-independent viscosity (Fig. 2A). Phase-separated bilayers such as DPPC:DOPC (1:1), which have solid domains coexisting with liquid-disordered continuous phase (see inset of 2B) [51 53, and the diblock-copolymer membranes shear thin, i.e., their viscosity decreases with increasing shear rate (Fig. 2B-C and Appendix Section 3).

Intriguingly, measurements at the same field amplitude 
but different frequencies revealed an apparent increase of viscosity with frequency (Fig. 2D). More precisely, the viscosity shows an initial linear increase with frequency and a plateau above few $\mathrm{kHz}$ (Fig. 2E). The behavior is observed with all studied compositions. Viscosity values in the literature obtained by methods that do not involve electric fields (Appendix Table II) are at least a factor of 10 smaller than the plateau-value of the viscosity but comparable to the zero-frequency limit obtained from the linear extrapolation at low frequencies. We hypothesize that the zero-frequency viscosity is representative for the viscosity of the membrane in the absence of electric field. As a test, we analyzed the vesicle relaxation back to its equilibrium shape, after the field is turned off (Fig. 2F). That analysis, however, is complicated by the fact that unlike the initial elongation upon application of the field, the relaxation is driven by and thus depends on the membrane tension. In order to leave the membrane viscosity as the only fitting parameter for the relaxation curve, the tension needs to be independently determined. In this experiment, the tension was obtained from the analysis of the equilibrium thermally-excited membrane undulations (flickering spectroscopy) in the absence of electric field before the electrodeformation experiment. The fit of the relaxation curve with Eq. 1 1 yielded viscosity $21.1 \pm 29.4$ nPa.s.m. The large error is due to the uncertainty in the tension. The viscosity value is close to the zero-frequency limit of the viscosity obtained from the frequency sweep ( $9.73 \pm 5.80$ nPa.s.m) for SOPC. We adopt the frequency sweep to determine the initial linear dependence of the viscosity and its "no-field" limit.

To summarize, the method involves measuring apparent viscosities at different frequencies in the range 0.1-1 $\mathrm{kHz}$ and extrapolating to zero-frequency (as in Fig2D) to obtain the value of the viscosity in the absence of electric field. Electric field of $8 \mathrm{kV} / \mathrm{m}$ (strain rate $50 \mathrm{~s}^{-1}$ ) produces a good range of data in the initial linear deformation regime. Measurement and data analysis of one vesicle typically takes about $10 \mathrm{~min}$. We analyze 10-50 vesicles per viscosity value. The viscosity obtained for 14 different bilayer compositions is reported in Table I. The values are in the range reported in previous studies (Appendix Table II). For example, the bilayer viscosity for SOPC is in good agreement with previously reported values from 3 to $13 \mathrm{nPa}$.s.m [26, 27, 54].

\section{Viscosity correlation with lipid diffusivity, membrane composition and thickness.}

Since mobility of lipids or domains is often used to asses membrane fluidity [12 14, we have compared our results for membrane viscosity with measurements of the diffusion coefficient of a lipid dye (DiI-C18) using Fluorescence Correlation Spectroscopy (FCS) in the same ternary system [55]. The diffusion coefficient scales inversely with surface viscosity (Fig. 3), a trend expected from the Saffman-Delbrück's model [56], $D=$
TABLE I. Membrane viscosity and bending rigidity for various bilayer systems at $25.0^{\circ} \mathrm{C}$ and $E_{0}=8 \mathrm{kV} / \mathrm{m}$ (strain rate $\dot{\gamma}=50 \mathrm{~s}^{-1}$ ).

\begin{tabular}{lll}
\hline Composition & $\begin{array}{l}\text { Viscosity } \\
(\text { nPa.s.m })\end{array}$ & $\begin{array}{l}\text { Bending Rigidity } \\
\left(k_{B} T\right)\end{array}$ \\
\hline DOPC & $4.11 \pm 2.63$ & $22.2 \pm 2.0$ \\
OMPC & $7.73 \pm 3.09$ & $27.1 \pm 2.6$ \\
POPC & $9.32 \pm 5.95$ & $27.8 \pm 2.3$ \\
SOPC & $9.73 \pm 5.80$ & $30.1 \pm 3.1$ \\
DOPC:Chol & $7.00 \pm 4.77$ & $27.8 \pm 4.6$ \\
DPPC:DOPC:Chol $(1: 1: 1)$ & $17.7 \pm 3.06$ & $72.0 \pm 8.4$ \\
DPPC:DOPC:Chol $(1: 1: 2)$ & $15.4 \pm 2.40$ & $69.2 \pm 7.9$ \\
DPPC:Chol $(1: 1)^{\text {PBd }} 13-b-\mathrm{PEO}_{11}$ & $56.4 \pm 4.63$ & $121.3 \pm 11.0$ \\
PBd $_{22}-b-\mathrm{PEO}_{14}$ & $14.4 \pm 4.40$ & $17.1 \pm 1.5$ \\
PBd $_{33}-b-\mathrm{PEO}_{20}$ & $686 \pm 51.0$ & $31.0 \pm 5.1$ \\
PBd $_{46}-b-\mathrm{PEO}_{24}$ & $2890 \pm 670$ & $54.4 \pm 6.4$ \\
PBd $_{54}-b-\mathrm{PEO}_{29}$ & $20600 \pm 4700$ & $\mathrm{NA}$ \\
PBd $_{120}-b-\mathrm{PEO}_{78}$ & $46700 \pm 900$ & $154.0 \pm 16.0$ \\
\hline
\end{tabular}

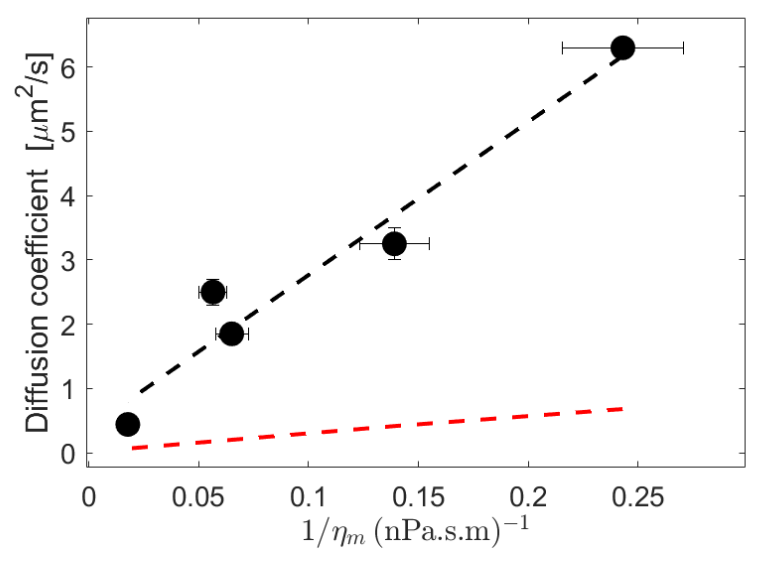

FIG. 3. Membrane viscosity as a function of diffusivity values obtained with FCS Scherfeld et al. 55. for membrane compositions DOPC, DOPC:Chol (1:1), DPPC:DOPC:Chol (1:1:2), DPPC:DOPC:Chol (1:1:1) and DPPC:Chol (1:1). Values of the diffusion coefficient are listed in the SI. The red dashed line corresponds to the prediction from the SaffmanDelbrück's model with probe radius $r=0.5 \mathrm{~nm}$. The black dashed line is a linear fit with intercept $0.383 \mu^{2} \mathrm{~m} / \mathrm{s}$ and slope $23.83 \mu \mathrm{m}^{3}$. $\mathrm{mPa}$

$k_{B} T /\left(4 \pi \eta_{m}\right)\left(\log \left(\eta_{m} /(\eta r)\right)-0.5772\right)$. However, there is a quantitative disagreement: using lipid dye radius $r=$ $0.5 \mathrm{~nm}$ (comparable to the radius of DOPC estimated from the area per lipid head, 67.4-75.4 $\AA^{2}$ [57-62]) the Saffman-Delbrück's equation predicts much lower diffusivities. These results suggest that while increasing viscosity does correlate with decreasing diffusivity, it is not trivial to relate membrane viscosity and the diffusion constant because diffusion of molecular probes is sensitive to 
A)

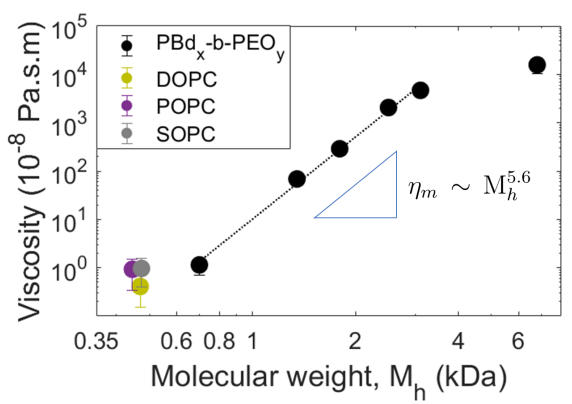

B)

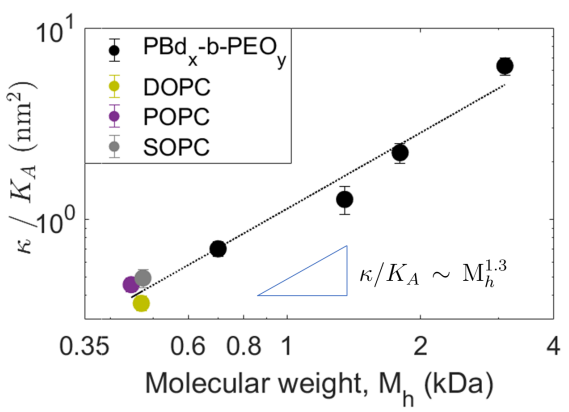

C)

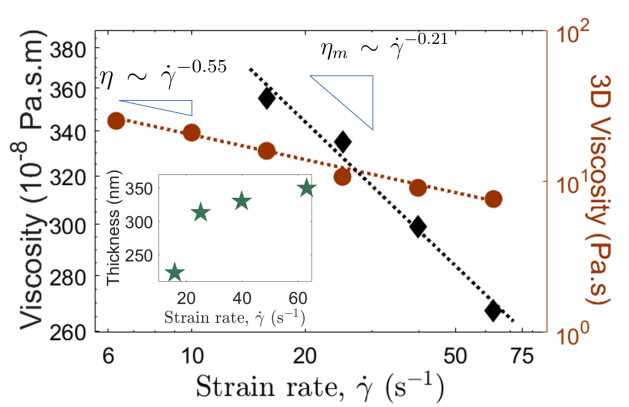

FIG. 4. A) Viscosity of $\mathrm{PBd}_{x}-b-\mathrm{PEO}_{y}$ bilayers as a function of the $M_{h}$ of the hydrophobic part (PBd). Viscosities of phospholipids are also shown for comparison. B) Bending rigidity of $\mathrm{PBd}_{x}-b-\mathrm{PEO}_{y}$ and phospholipids bilayer membranes as a function of molecular weight. The power law obtained is $\kappa / K_{A} \sim M_{h}^{1.3}$. C) Comparison of the shear-thinning of the 2D and $3 \mathrm{D}$ viscosities of $\mathrm{PBd}_{33}-b-\mathrm{PEO}_{20}$. The inset shows the membrane thickness obtained from $\eta_{m} / \eta=h$.

the probe itself as well as the bilayer structure [11, 14, 21. However, the empirically obtained dependence in Fig. 3 . could presumably be used to roughly deduce membrane viscosity from molecular diffusivity measurements.

The viscosity values complied in Table I highlight that in pure lipid systems membrane viscosity decreases with the number of unsaturated bonds in the hydrophobic tail. POPC, OMPC and SOPC have a single unsaturated bond, while DOPC has two double bonds in the hydrophobic tails. DOPC bilayers exhibit much smaller viscosity than the other single unsaturated lipid bilayers. In the mixed systems, adding DPPC or/and cholesterol to DOPC increases the viscosity. Adding cholesterol (molar ratio 1:1) to DOPC increases the bilayer viscosity as cholesterol increases the packing of the liquid disordered $\left(L_{d}\right)$ phase. For liquid ordered $\left(L_{o}\right)$ phase, such as DPPC:Chol (1:1), the bilayer viscosity is much higher due to the tight packing provided by saturated acyl chains. The effect is less pronounced in the ternary $L_{o}$ system DOPC:DPPC:Chol (1:1:2) and DOPC:DPPC:Chol (1:1:2). For a phase-separated system of coexisting solid and fluid phases DOPC:DPPC (1:1), see Fig. 2B, the viscosity also increases relative to pure DOPC. The increase agrees with an estimate of the viscosity of a $2 \mathrm{D}$ suspension, $\eta_{\text {eff }}=\eta_{D O P C}(1+2 \phi)$, where $\phi \sim 0.4$ is the fraction of the solid phase [63.

Using our method we were also able to examine the commonly assumed relation between the $2 \mathrm{D}\left(\eta_{m}\right)$ and $3 \mathrm{D}$ viscosities $\left(\eta_{3 D}\right), \eta_{m}=\eta_{3 D} h$, where $h$ is the membrane thickness. The thickness of diblock-polymer bilayers increases with the molecular weight of the hydrophobic part, $h \sim M_{h}^{n}$ 64, 65], where $n$ lies within the theoretical bounds of 0.5 (random Gaussian coil) and 1 (full stretch). The polymers' molecular weight varies in a wide range (1$8 \mathrm{kDa}$ ) thereby resulting in bilayers with greater range of thicknesses, unlike lipids. The diblock polymers showed membrane viscosity spanning four orders of magnitude range, from $14 \mathrm{nPa}$.s.m to $157000 \mathrm{nPa}$.s.m. The lowest molecular-weight polymer membrane exhibits a viscosity similar to that of POPC. The membrane viscosity in Fig.
4 A does follow a power-law dependence on $M_{h}$, but the power 5.6 is much larger than the expected range $0.5-1$. In contrast, as seen from Fig. $4 \mathrm{~B}$, the bending rigidity follows a power-law consistent with the expected $\kappa \sim M_{h}^{2 n}$, since $\kappa / K_{A} \sim h^{2}$, where $K_{A}$ is the area-compressibility modulus [66]. The $K_{A}$ value is relatively insensitive to the molecular weight; its value for the lipids was taken to be $250 \mathrm{mN} / \mathrm{m}$ and for the polymer membranes - 100 $\mathrm{mN} / \mathrm{m}$ 67, 68. The polymer 3D viscosity $\eta$, however, varies with the polymer molecular weight and this may be the source of the unexpectedly higher exponent in the power-law dependence of $\eta_{\mathrm{m}}$ on $M_{h}$. Comparison of the dependence on the strain-rate of the $2 \mathrm{D}$ and $3 \mathrm{D}$ viscosities (Fig. 4C and Appendix Section S4) shows that in three dimensions the shear-thinning is more pronounced. This suggests that the polymer confinement into a bilayer impedes the microstructure dynamics responsible for the shear thinning. Intriguingly, taking the ratio of the $2 \mathrm{D}$ and $3 \mathrm{D}$ viscosities yields a thickness $h$ ranging from 200 to $350 \mathrm{~nm}$ much larger than typical thickness measured with cryo-TEM [69]. The disparity might arise from the thermally-driven membrane undulations, which result in interface roughness that is in the order of $100 \mathrm{~nm}$ for a tension-free membrane and $20 k_{B} T$ bending modulus. Thus the effective membrane thickness would be larger for 'fuzzy' membranes.

\section{DISCUSSION AND CONCLUSIONS}

We developed a method to measure the shear viscosity of the two-dimensional bilayer fluid using the transient deformation of a giant vesicle. The approach is inspired by the interfacial rheology measurements using deformation of droplets and capsules [70. The "vesiclerheometer" enabled the collection of an unprecedented amount of data, which provided valuable insights into the rheology of bilayer membranes and lead to two new intriguing findings that raise more questions.

First, bilayer membranes can behave as non- 
Newtonian fluids. Phase-separated bilayers are the 2D analogs of 3D dispersed systems such as emulsions and suspensions, which exhibit shear thinning behavior due to microstructure reorganization and relaxation; the domain dynamics in the phase-separated bilayers is likely the source of the shear thinning dynamics. Likewise, the shear thinning viscosity of the diblock-copolymer membranes likely originates from polymer stretching and relaxation on a time scale comparable to the shearing.

Second, membrane viscosity increases with field frequency reaching a plateau above few $\mathrm{kHz}$. The zerofrequency limit of the viscosity is comparable to the one measured in the absence of electric field by other methods, thus the frequency effect is not related to the electric field amplitude. We hypothesize that the phenomenon is due to membrane electrical polarization. In an applied electric field, ions brought by conduction accumulate at the membrane surfaces, because the bilayer is impermeable to ions (Fig. 5). At low frequencies,

A)
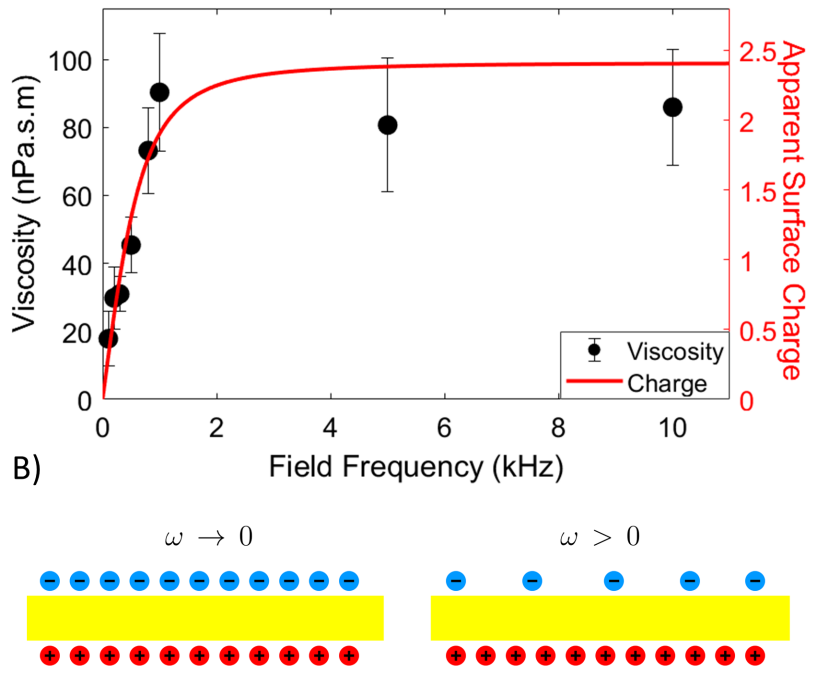

FIG. 5. A) Membrane viscosity increases with field frequency. Data for SOPC, $E_{0}=8 \mathrm{kV} / \mathrm{m}$. Same behavior is exhibited by the imbalance in the surface charges accumulated at the opposing membrane surfaces computed from Eq. 7 in SI. B) Schematic illustration of the membrane (yellow) and free charges (blue and red) under an external electric field. The membrane capacitor is fully charged at low frequencies but becomes short-circuited at higher frequencies giving rise to an imbalance of the induced charge.

$\omega \ll \omega_{c} \sim \lambda_{\mathrm{v}} / R C_{m}$, where $C_{m}$ is the membrane capacitance and $\lambda_{\mathrm{v}}$ is the interior solution conductivity, the membrane capacitor is fully charged and the net charge of the membrane (sum of the accumulated charge on the two surfaces) is zero. As the frequency increases, $\omega \gtrsim \omega_{c}$, the capacitor becomes short-circuited and draws current. This gives rise to an imbalance in the induced surface charge on the the opposite membrane surfaces; this imbalance (equivalent to an apparent membrane charge) correlates remarkably well with the viscosity frequency dependence, see Fig. 5. The only study of the effect of surface charge on the viscosity of bilayers, using mixtures of charged and neutral surfactants, has reported a significant increase 71]. The bilayers in our study are made of charge-neutral lipids, however, an apparent surface charge originates from excess mobile charges (ions) at the membrane surfaces. The mechanisms underlying the viscosity dependence on strain-rate and field frequency need further systematic exploration.

The ease of implementation, high-throughput, minimal experimental equipment and effort as well as robustness make this technique easy to adopt in every lab. The method can also be applied to obtain interfacial properties of lipid monolayers using deformation of droplets. We envision that our approach will become a standard tool for characterization of membrane fluidity that will help address questions of biological and engineering importance such as mechanics of excitable cells and synthetic cell design.

\section{MATERIALS AND METHODS}

\section{Vesicle Preparation}

Giant unilamellar vesicles (GUVs) are formed from lipids and polymer such as palmitoyloleoylphosphatidylcholine (POPC), dioleoylphosphatidylcholine (DOPC), oleoylmyristoylphosphatidylcholine (OMPC), cholesterol (Chol), stearoyloleoylphosphatdylcholine (SOPC), dipalmitoylphosphatidylcholine (DPPC) and poly(butadiene)- $b$-poly(ethylene oxide) diblock copolymers, $\mathrm{PBd}_{x}-b-\mathrm{PEO}_{y}$. The lipids and diblock copolymer were purchased from Avanti Polar Lipids (Alabaster, AL) and Polymer Source Inc. (Montreal, Canada), respectively. The multi-component vesicles made of DOPC:DPPC:Chol were fluorescently marked with 0.1 mol\% of Liss Rhod PE. The lipid vesicles were produced using the electroformation method 72]. The stock solutions of $12 \mathrm{mM}$ lipid in choloroform are diluted to 5 $\mathrm{mM}$ from which $10 \mu \mathrm{l}$ of the solution is spread on the conductive sides of the ITO slides (Delta technologies, USA). The slides are stored in vacuum for 2-4 hours to evaporate all the organic solvents. The two slides are then sandwiched with a $2 \mathrm{~mm}$ thick teflon spacer and the electroformation chamber is filled with $40 \mathrm{mM}$ sucrose solution in $0.3 \mathrm{mM}$ of $\mathrm{NaCl}$. The chamber is connected to a signal generator (Agilent, USA) for 2 hours at $50 \mathrm{~Hz}$ and voltage $1.5 \mathrm{~V}$ at $60^{\circ} \mathrm{C}$, which ensures that all lipids are above their main phase transition temperatures. The harvested vesicles are diluted in isotonic glucose solution without salt. 3 independent GUV batches for every lipid composition were analyzed. Polymer vesicles were produced from spontaneous swelling method. Initially, $50 \mu \mathrm{l}$ of $6-10 \mathrm{mg} / \mathrm{ml}$ (in chloroform) polymer solution was dissolved in 200-300 $\mu \mathrm{l}$ of chloroform in a $20 \mathrm{ml}$ vial. Polymer films were formed from evaporation 
by blowing with a nitrogen stream while swirling the solution inside. Afterwards, the vials were dried under vacuum for 2-4 hours. The polymer films were hydrated in the suspending solutions $(40 \mathrm{mM}$ sucrose solution in $0.3 \mathrm{mM} \mathrm{NaCl}$ ) and placed at $60 \mathrm{C}$ in an oven for 18-24 hours.

\section{Electrodeformation}

The electrodeformation experiments are conducted in the electrofusion chamber (Eppendorf, Germany). The chamber is made from Teflon with two $92 \mu \mathrm{m}$ cylindrical parallel electrodes $500 \mu \mathrm{m}$ apart. The field is applied using a function generator (Agilent 3320A, USA). The function generator is controlled using a custom built MATLAB (Mathworks, USA) progam. This gives a precise control over the strength and duration of applied electric fields [73].

\section{Optical microscopy and imaging}

The vesicles are visualized using a phase contrast microscope (A1 Axio Observer, Zeiss, Germany) with 63x objective $0.75 \mathrm{NA}$ (air). Imaging is performed using Photron SA1.1 camera. The image acquisition rate for electrodeformation recordings is kept to a constant of 500-2000 fps for lipid vesicles and 60-200 fps for polymer vesicles and the shutter speed is fixed to 500 $\mu \mathrm{s}$. The time evolution of the vesicle is analyzed using a home-made image analysis software. The software uses a Fourier series to fit around the vesicle contour, $r_{s}=\sum_{n=0}^{\infty} \alpha_{n} \cos (n \theta)+\beta_{n} \sin (n \theta)$. The second mode in the series is used to determine the major $(a)$ and minor axis $(b)$ of the deformed vesicles to evaluate $\nu=\frac{a}{b}=\left(1+\alpha_{2}\right) /\left(1-\alpha_{2}\right)$. The confocal imaging was performed with Leica TCS SP8 scanning confocal microscope using a HC PL APO 40x/ NA 1.3 (oil) objective. The pinhole size during the experiment was fixed to 1 AU (Airy units). The dye was excited with a $561 \mathrm{~nm}$ laser (diode-pumped solid-state laser) with $1.61 \%$ (laser intensity) HyD3 detector (hybrid).

\section{Bending rigidity and tension measurements}

Flickering spectroscopy is a popular technique to extract out membrane rigidity and tension due to its nonintrusive nature and well developed statistical analysis criteria. The details of the technique are given in [74-76. Essentially, a time series of fluctuating vesicle contours is recorded on the focal plane. The quasicircular contour is represented in Fourier modes, $r(\phi)=$ $R\left(1+\sum_{q} u_{q}(t) \exp (\mathrm{i} q \phi)\right)$. The fluctuating amplitudes $u_{q}$ have mean square amplitude dependence on the membrane bending rigidity $\kappa$ and the tension $\sigma,\left\langle\left|u_{q}\right|^{2}\right\rangle \sim$
$\frac{k_{B} T}{\kappa\left(q^{3}+\bar{\sigma} q\right)}$, where $k_{B} T$ is the thermal energy $\left(\mathrm{k}_{B}\right.$ is the Boltzmann constant and $\mathrm{T}$ is the temperature), and $\bar{\sigma}=\sigma R^{2} / \kappa$. The integration time effect of the camera was reduced by acquiring images at a low shutter speed of $100-200 \mu \mathrm{s}$. At least 5000 images were obtained for each vesicle for good statistics.

\section{ACKNOWLEDGMENS}

P.M.V and H.A.F acknowledge financial support by NIGMS award 1R01GM140461. This research was also supported in part by the National Science Foundation under Grant NSF PHY-1748958. 
Appendix A: Compendium of published values for membrane viscosity

see Table II 


\section{Appendix B: Shape evolution of quasi-spherical vesicle in a uniform electric field}

\section{Theoretical model}

A)

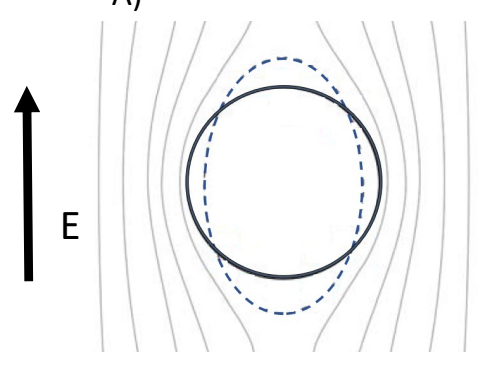

B)

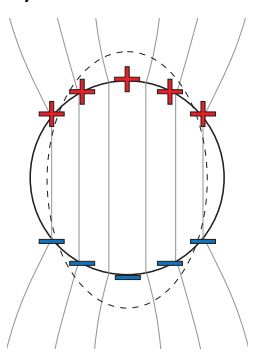

C)

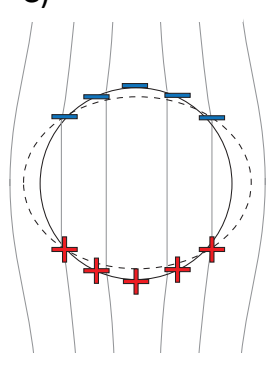

D)

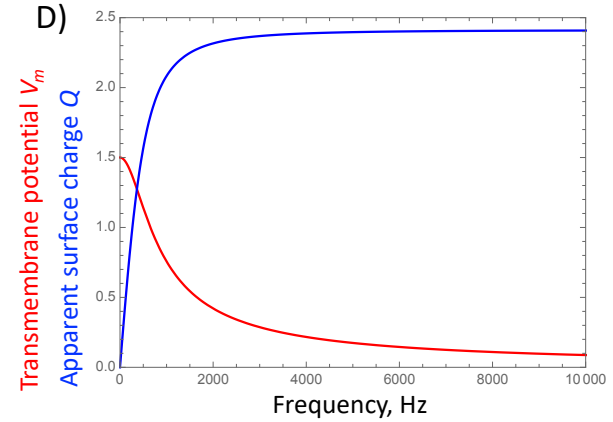

FIG. 6. (A)-(C) Physical mechanisms of the frequency-dependent membrane polarization and vesicle dipole in an applied uniform AC field. The lines correspond to constant electric field. Upon application of an external electric field, charges accumulate on the two sides of the membrane setting up a potential difference, i.e., the membrane acts as a capacitor. (A) At low frequencies, $\omega \ll \omega_{c}$, the membrane capacitor is fully charged, the induced charge density on the two membrane surfaces is the same but of opposite sign. (B) and (C) At intermediate frequencies, $\omega>\omega_{c}$, it is short-circuited and there is charge imbalance between the inner and outer membrane surfaces $Q=\varepsilon E_{0} Q_{0} \cos \theta$. (B) If the enclosed solution is more conducting than the suspending medium, $\Lambda>1$, vesicle is pulled into an prolate ellipsoid. (C) The polarization is reversed in the opposite case $\Lambda<1$ and the vesicle deforms into an oblate ellipsoid. (D) Variation with frequency of the transmembrane potential (red) and apparent charge at the pole (blue).

Let us consider a vesicle made of a charge-free lipid bilayer membrane with bending rigidity $\kappa$, tension $\sigma_{\text {eq }}$, capacitance $C_{\mathrm{m}}$. The vesicle is suspended in a solution with conductivity $\sigma_{\mathrm{w}}$ and permittivity $\varepsilon_{\mathrm{w}}$, and filled with a different solution characterized by $\sigma_{\mathrm{v}}$ and $\varepsilon_{\mathrm{v}}$.

An axisymmetric stress, such as generated by uniform electric field or extensional flow, deforms the vesicle into a spheroid with symmetry axis aligned with the extensional axis. The spheroid aspect ratio is $\nu=a / b$, where $a$ is the length of the symmetry axis and $b$ is the length of the axis perpendicular to the symmetry axis. For small deformations, $\nu \lesssim 1.3$, the shape is well approximated by

$$
r_{s}(\theta)=R\left(1+\frac{s}{2}(1+3 \cos 2 \theta)\right)
$$

where $r_{s}$ is the position of the surface, $R$ is the initial radius of the vesicle, $s$ is the deformation parameter, and $\theta$ is the angle with the applied field direction; $\theta=0$ and $\pi / 2$ correspond to the pole and the equator, respectively. The ellipsoid aspect ratio is related to the deformation parameter by $\nu=(1+s) /(1-2 s)$.

The theory developed by Vlahovska et al. [45, 46, 50, 86] predicts that the deformation parameter evolution is given by the balance of imposed and membrane stresses

$$
\dot{s}=\frac{1}{32+23 \chi+16 \chi_{m}}\left(\frac{\varepsilon_{\mathrm{w}} E_{0}^{2} p^{\mathrm{el}}}{\eta}-24 s\left(6 \kappa+\sigma(s) R^{2}\right) \frac{1}{\eta R^{3}}\right)
$$

The AC field, $E(t)=E_{0} \sin (\omega t)$, generates an electric stress which has two components, a steady one $p^{\mathrm{el}}$ and an oscillatory one with frequency twice the applied one

$$
p=p^{\mathrm{el}}+p_{\omega}^{c} \cos (2 \omega t)+p_{\omega}^{s} \sin (2 \omega t)
$$

In the experiments typically $\bar{\omega} \gg 1$ and the oscillatory component only drives very small oscillations about the deformation induced by the steady stress component.

The steady electric stress is given by

$$
p^{\mathrm{el}}=2\left(1-P_{\mathrm{w}}^{r}\right)+\frac{1}{2} P_{\mathrm{w}}^{2}-2 S P_{\mathrm{v}}^{2}
$$


and the amplitudes of the unsteady stress are

$$
\begin{aligned}
& p_{\omega}^{c}=\frac{1}{2}\left(4\left(1-P_{\mathrm{w}}^{r}\right)-\left(P_{\mathrm{w}}^{i}\right)^{2}+\left(P_{\mathrm{w}}^{r}\right)^{2}-4 S\left(\left(P_{\mathrm{v}}^{i}\right)^{2}+\left(P_{\mathrm{v}}^{r}\right)^{2}\right)\right) \\
& p_{\omega}^{s}=2 P_{\mathrm{w}}^{i}-P_{\mathrm{w}}^{i} P_{\mathrm{w}}^{r}+4 S P_{\mathrm{v}}^{i} P_{\mathrm{v}}^{r}
\end{aligned}
$$

where

$$
\begin{aligned}
& P_{\mathrm{w}}=\frac{K_{\mathrm{w}}+K_{\mathrm{v}}\left(V_{m}-1\right)}{K_{\mathrm{v}}+2 K_{\mathrm{w}}}, \quad P_{\mathrm{v}}=\frac{K_{\mathrm{w}}\left(3-2 V_{m}\right)}{K_{\mathrm{v}}+2 K_{\mathrm{w}}}, \\
& V_{m}=\frac{3 K_{\mathrm{v}} K_{\mathrm{w}}}{2 K_{\mathrm{v}} K_{\mathrm{w}}+\mathrm{i} C_{\mathrm{m}}\left(K_{\mathrm{v}}+2 K_{\mathrm{w}}\right) \bar{\omega}}
\end{aligned}
$$

Here $\bar{\omega}=\omega \varepsilon_{\mathrm{w}} / \sigma_{\mathrm{w}}$ and $\bar{C}_{\mathrm{m}}=C_{\mathrm{m}} R / \varepsilon_{\mathrm{w}}$ are the dimensionless frequency and membrane capacitance. $K_{\mathrm{v}}=1+\mathrm{i} \bar{\omega}$ and $K_{\mathrm{w}}=\Lambda+\mathrm{i} \bar{\omega} S$ are the dimensionless complex permittivities. $S=\varepsilon_{\mathrm{v}} / \varepsilon_{\mathrm{w}}$ and $\Lambda=\sigma_{\mathrm{v}} / \sigma_{\mathrm{w}}$ are the ratios of permittivities and conductivities of the fluids interior and exterior to the vesicle. $P^{r}$ and $P^{i}$ denote the real and imaginary part of $P$, and $P^{2}=P P^{*}$, where the superscript ${ }^{*}$ denotes complex conjugate. The electric stress in DC field is obtained by setting $\bar{\omega}=0$ and the electric field amplitude to $E_{0} \sqrt{2}$.

Typically, both the inner and outer fluids are aqueous solutions with similar permittivities, $\varepsilon_{\mathrm{v}} \approx \varepsilon_{\mathrm{w}}=\varepsilon$, hence $S$ can be set to 1. In this case Eq. B3 reduces to

$$
p^{\mathrm{el}}=\frac{9\left[\bar{\omega}^{2}\left(\bar{C}_{\mathrm{m}}^{2}(\Lambda+2)^{2}(\Lambda-1)(\Lambda+3)+2 \bar{C}_{\mathrm{m}} \Lambda\left(\Lambda^{2}+\Lambda-2\right)+9 \Lambda^{2}\right)+\Lambda^{2}(\Lambda+2)^{2}\right]}{2\left((\Lambda+2)^{2}+9 \bar{\omega}^{2}\right)\left(\bar{C}_{\mathrm{m}}^{2}(\Lambda+2)^{2} \bar{\omega}^{2}+4 \Lambda^{2}\right)},
$$

where $\bar{\sigma}=\sigma R^{2} / \kappa$. At low frequencies, $\bar{\omega} \rightarrow 0$, the membrane capacitor is fully charged, and $p^{\text {el }}=9 / 16$ and we obtain Equation 1 in the main text.

The imbalance between the induced charge of the two membrane surfaces is $Q=\varepsilon E_{0} Q_{0} \cos \theta$, where the maximum charge is

$$
Q_{0}=\frac{3 \bar{\omega} C_{m}(\Lambda-1)(\Lambda+2)}{\left[\left((2+\Lambda)^{2}+9 \bar{\omega}^{2}\right)\left(4 \Lambda^{2}+C_{m}^{2} \bar{\omega}^{2}(2+\Lambda)^{2}\right)\right]^{1 / 2}}
$$

At low frequencies, $\bar{\omega} \rightarrow 0$ the charge imbalance vanishes.

\section{Linear approximation of the evolution equation}

Assuming constant tension, Eq. 1 in the main text can be integrated to yield

$$
\nu(t)=1+\frac{p R}{24 \sigma}\left(1-\exp \left(-\frac{24 \sigma}{\eta R\left(55+16 \chi_{m}\right)} t\right)\right)
$$

If

$$
\frac{24 \sigma t}{\eta R\left(55+16 \chi_{m}\right)} \ll 1
$$

the exponent is expanded in Taylor series to yield the linear evolution, Eq. 2 in the main text.

The time limit for the linear approximation can be estimated by comparing the linear and quadratic terms in the Taylor series of the exponential term in Eq. B8. The Taylor series of the exponential function is

$$
\exp (-c t)=1-c t+\frac{(c t)^{2}}{2}+\text { h.o.t. } \quad \text { where for Eq. } 11 \quad c=\frac{24 \sigma}{\eta R\left(55+16 \chi_{m}\right)}
$$

It shows that the quadratic correction becomes comparable to the linear term when

$$
c t=\frac{(c t)^{2}}{2} \Longrightarrow t=\frac{2}{c}
$$

which gives the estimate for the time up to which the linear approximation is reasonable

$$
\frac{t}{t_{\mathrm{d}}}=\frac{\varepsilon E_{0}^{2} R\left(55+16 \chi_{m}\right)}{12 \sigma}
$$




\section{Appendix C: Additional data}

\section{Batch reproducibility for homogeneous (DOPC) and mixed membrane compositions (DOPC:DPPC:Chol} $(1: 1: 1))$

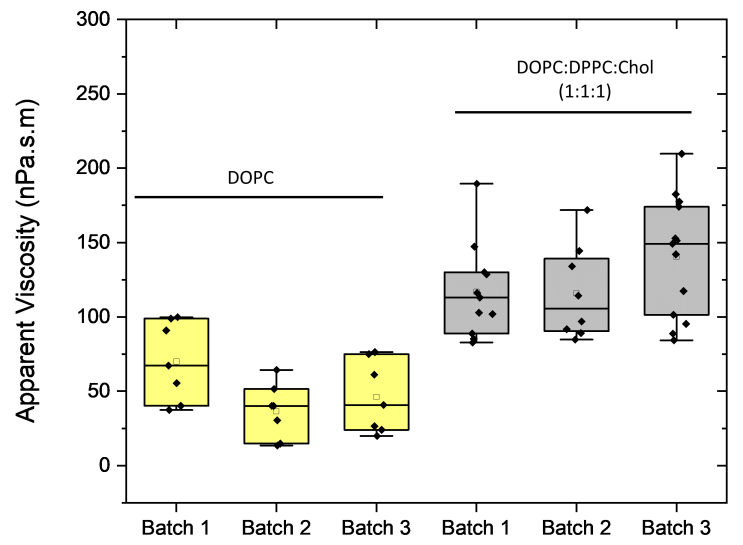

FIG. 7. Method reproducibility for the same system across different batches: Membrane viscosity for DOPC and DOPC:DPPC:Chol (1:1:1) for three different batches of prepared vesicles. The applied frequency and field strength are 1 $\mathrm{kHz}$ and $10 \mathrm{kV} / \mathrm{m}$ for respectively. For DOPC:DPPC:Chol (1:1:1), the applied frequency is $2 \mathrm{kHz}$ and and applied field strength is $6 \mathrm{kV} / \mathrm{m}$. The solid symbols show measurements on individual vesicles. The box-plot represents the standardized distribution of data based on five numbers minimum value, first quartile (Q1), median, third quartile (Q3), and maximum value. The open square represents the mean value..

Figure 7 shows the box plot presentation for apparent membrane viscosity values obtained for the same system across three different batches of vesicles prepared from DOPC and DOPC:DPPC:Chol (1:1:1). The zero charge or frequency membrane viscosity data is given in the main text.

\section{Deformation curves of bilayers at different field strength and frequency}

Figure 8A represents deformation curves of POPC vesicles at different field strength $(6-10 \mathrm{kV} / \mathrm{m})$ at frequency 1 $\mathrm{kHz}$. In Figure $8 \mathrm{~B}$ the data is re-plotted again in rescaled time with $t_{\mathrm{d}}$. The inverse of $t_{\mathrm{d}}$ can be expressed as shear rate, $\dot{\gamma}$ which in this case ranges from, $\dot{\gamma} \sim 10-100 \mathrm{~s}^{-1}$. The collapse of the data on single curve indicates that the deformation rate of POPC bilayers are not affected at a given shear rate and they exhibit Newtonian rheology.

Eq. 2 in the main text shows that the slope depends on $t_{\mathrm{d}}$, which depends on the field amplitude $E_{0}$, and $p^{\mathrm{el}}$, which depends on frequency. Hence to isolate the viscosity, one needs to plot the deformation data as a function of time rescaled as $t / t_{\mathrm{d}} p^{\mathrm{el}}$, see Figure 9 .

\section{Newtonian and Non- Newtonian bilayers}

Figure 10 shows flow curve experiment (membrane viscosity vs imposed shear rate) for different compositions. Unsaturated phospholipids, for example DOPC in 10A and B, are known to assemble into bilayers in the liquid disordered, $L_{d}$ phase. Our data shows that at different shear rate, $\dot{\gamma} \sim 10-1000 \mathrm{~s}^{-1}$, or E- field ranging from 5 -40 $\mathrm{kV} / \mathrm{m}$, the membrane viscosity of $L_{d}$ bilayers remains unchanged. Therefore, DOPC bilayers are 2D Newtonian behavior. In the next case, see $10 \mathrm{C}$, we analyze, fully saturated lipids, DPPC, mixture with Cholesterol at 1:1 molar ratio. DPPC has a phase transition temperature temperature at $314 \mathrm{~K}$. Adding Cholesterol to the bilayers results in the disruption of the gel or solid phase of the bilayer to $L_{o}$ state which has a larger extent of orientational order than $L_{d}$. Our results show that $L_{o}$ are also Newtonian in nature. Next we move to mixed systems exhibiting phase separation. 1:1 DOPC:DPPC demonstrated phase separation with intricate network of finger-like domains indicative of gel or solid phase. Interestingly, the flow curve experiments 10D and E, show Non- Newtonian characteristics with shear thinning behavior (undergoing flow with a decreasing viscosity with increasing shear rate). Polymer vesicles assembled from di-block copoylmers such as $\mathrm{PBd}_{x}-b-\mathrm{PEO}_{y}$ also show a shear thinning behaviour with increasing 
A)

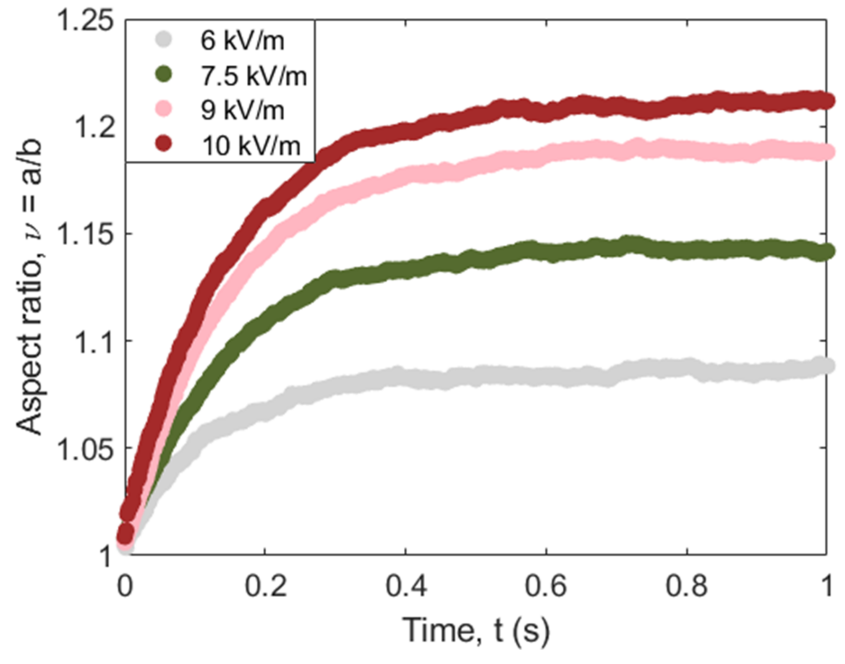

B)

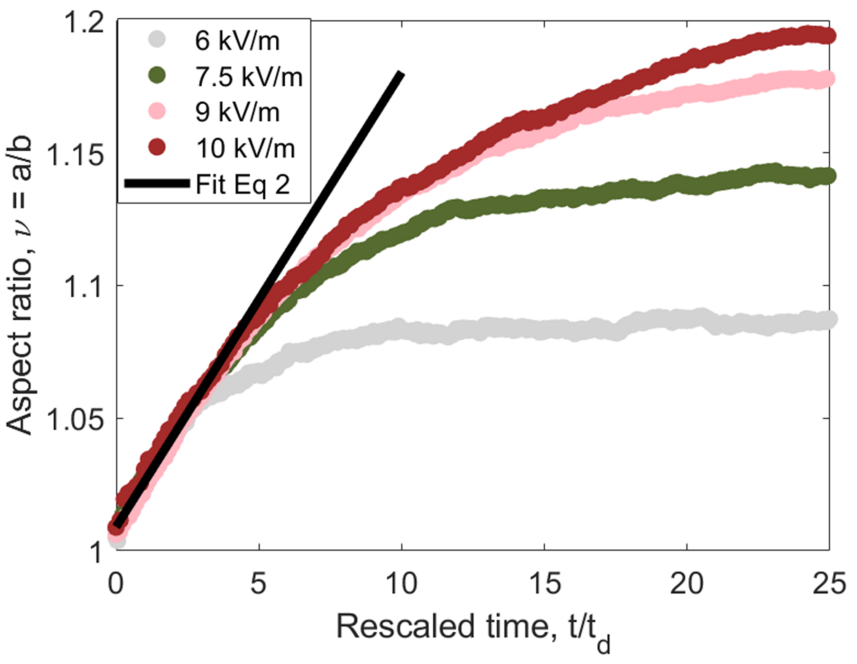

FIG. 8. (A) Deformation curves for a POPC vesicle $(\mathrm{R}=30.1 \mu \mathrm{m})$ exposed to fields of different amplitudes (at $1 \mathrm{kHz})$. (B) The initial slope of the data in (A) re-plotted as a function of the re-scaled time $t / t_{\mathrm{d}}$ yields an apparent membrane viscosity $\eta_{m}=2.63 \pm 0.41 \times 10^{-7}$ Pa.s.m.

A)

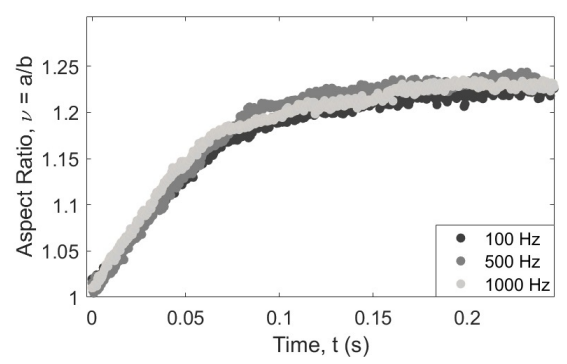

B)

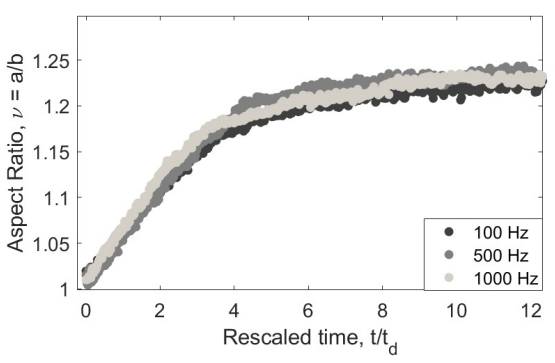

C)

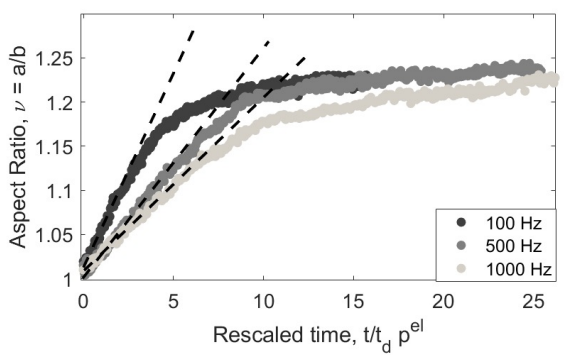

FIG. 9. (A) Deformation curves for a POPC vesicle $(\mathrm{R}=14.7 \mu \mathrm{m})$ exposed to fields of different frequency but same field amplitude $E_{0}=8 \mathrm{kV} / \mathrm{m}$. (B) The initial slope of the data in (A) re-plotted as a function of the re-scaled time $t / t_{\mathrm{d}}$, see Figure 9 The electric stress $p^{\mathrm{el}}$ increases with frequency but the slope in (B) remains the same indicating that apparent surface viscosity also increases. (C) Indeed, when data are plotted vs $t / t_{\mathrm{d}} p^{\mathrm{el}}$ the slope decreases with increasing frequency yielding the frequency dependence of the apparent viscosity. Extrapolation to zero frequency gives the membrane viscosity

shear rate. $10 \mathrm{~F}$ and $\mathrm{G}$ gives two examples of $\mathrm{PBd}_{x}-b-\mathrm{PEO}_{y}$ bilayers where the higher $M_{w}$ polymersomes, $\mathrm{PBd}_{33}-b$ $\mathrm{PEO}_{20}$, show a much stronger shear thinning behaviour. The viscosity of $\mathrm{PBd}_{33}-b-\mathrm{PEO}_{20}$ decreases by a $100 \%$ from $622 \pm 70 \times 10^{-8}$ Pa.s.m to $311 \pm 62 \times 10^{-8}$ Pa.s.m between shear rate $\dot{\gamma} \sim 10-1000 \mathrm{~s}^{-1}$. $\mathrm{PBd}_{13}-b-\mathrm{PEO}_{11}$ viscosity decreases from $8.72 \pm 2.22 \times 10^{-8}$ Pa.s.m to $5.6 \pm 0.5 \times 10^{-8}$ Pa.s.m between similar shear rates.

\section{Bending rigidity values from Flickering Spectroscopy and capacitance measurements for electrodeformation method}

The method for flickering spectroscopy is detailed in [75, 76. Here, we summarize the electrodeformation method to extract out membrane capacitance. The procedure follows the original approach developed by Salipante et al. [87. The vesicle shape morphology with conductivity ratio $\Lambda>1$ is always prolate. However, for $\Lambda<1$, the conductivity of the outer solution is higher than the vesicle solution and the aspect ratio/deformation parameter $s(\omega)$ is positive at low frequencies that is prolate shape. As the frequency increases, the vesicles becomes less prolate and adopts a spherical shape at a certain frequency. Above this critical frequency, the vesicles adopt an oblate shape. The critical 
A)

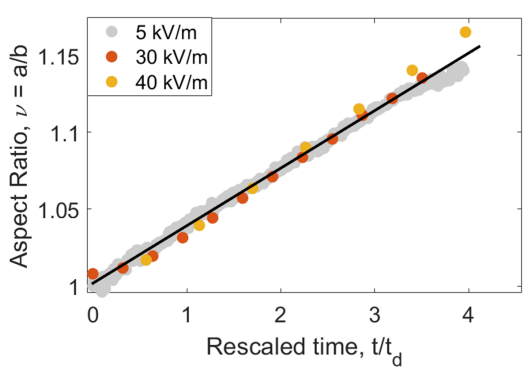

D)

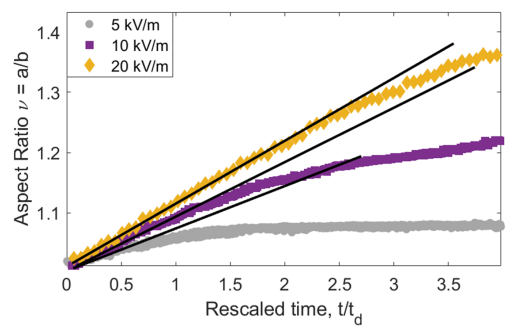

B)

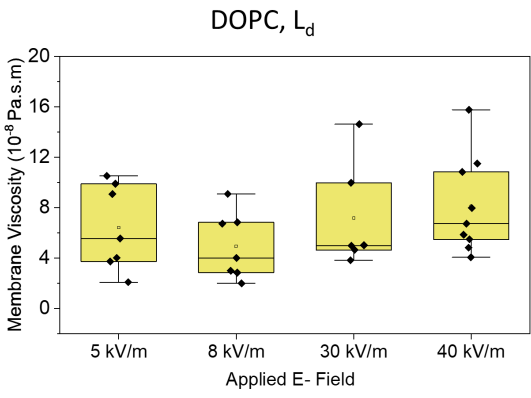

E)

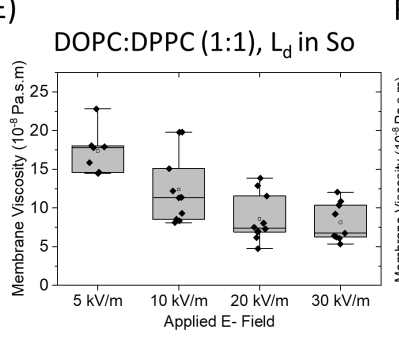

F)
C)
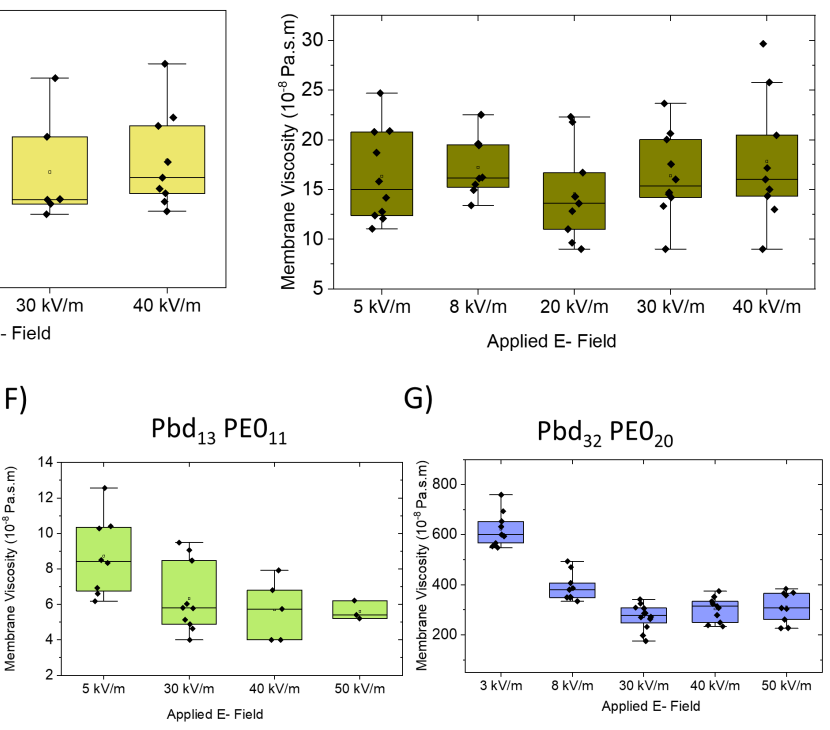

G)

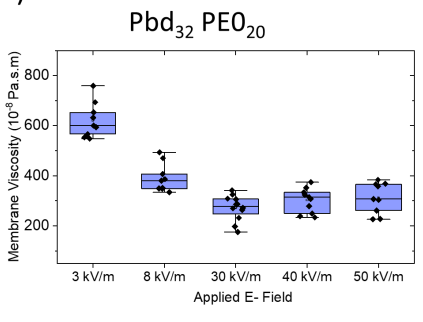

FIG. 10. Shear viscosity of different bilayer compositions at different electric field $(3-50 \mathrm{kV} / \mathrm{m})$ or shear rates $\dot{\gamma} \sim(1-1000) \mathrm{s}^{-1}$ at a fixed frequency $1 \mathrm{kHz}$. A) Deformation case of DOPC bilayer at different field strengths. The slope remains same regardless of the applied shear rate indicating 2D Newtonian fluids. B) Viscosity obtained at different field strengths for Newtonian cases B) DOPC C) DPPC:Chol (1:1) bilayers. D) Deformation case of DOPC:DPPC (1:1) bilayer at different field strength. The slope increases as the field strength or shear rate is increased. This behavior is represented by shear thinning fluids. B) Viscosity obtained at different field strengths for non-Newtonian cases E) DOPC:DPPC (1:1) F) $\left.\mathrm{PBd}_{13}-b-\mathrm{PEO}_{11} \mathrm{G}\right) \mathrm{PBd}_{32}-b-\mathrm{PEO}_{20}$.

frequency can be approximated as:

$$
\omega_{c}=\frac{\sigma_{\mathrm{v}}}{R C_{\mathrm{m}}} \frac{1}{\sqrt{(1-\Lambda)(3+\Lambda)}}
$$

Hence, the membrane capacitance can be determined from the experimentally measured critical frequency based on prolate-oblate transition with a frequency sweep [87. The measured bending rigidity and capacitance values are summarized in Table

\section{Appendix D: Rheology of block copolymer $\mathbf{P B d}_{33}-b-\mathbf{P E O}_{20}$.}

The copolymers $\mathrm{PBd}_{33}-b-\mathrm{PEO}_{20}$ was obtained from Polymer Source Inc. (Montreal, Canada). Figure 11A and $\mathrm{B}$ demonstrate the molecular formula and morphology of the melt at room temperature. The melt appears to be like a pasty wax. The shear rheology response was characterized using Anton MCR rheometer 302 for rheological testing. A parallel plate (PP $25 \mathrm{~mm}$ ) fixture was used for the measurements for low viscosity measurements. The parallel plate gap width was kept 0.7 to $\mathrm{mm}$. $0.5 \mathrm{gm}$ of the sample was loaded on the rheometer. The bulk shear viscosity, $\eta(\dot{\gamma})=\tau_{12} / \dot{\gamma}$, was determined from the measured shear stress, $\tau_{12}$, from imposed shear rates in the range from $\dot{\gamma}=10^{-2}-10^{2} \mathrm{~s}^{-1}$. As shown in Figure $11 \mathrm{C}$, the strain was chosen to be $1 \%$ to ensure that the sample was measured in linear viscoelastic regime. Figure 11D represents the viscosity at different shear rates. The block co polymer demonstrates consistent shear thinning, and total lack of zero-shear regime. The power law obtained in -0.51. This power law behavior is consistent with other published data in literature [88]. 
TABLE II. Membrane viscosity obtained using different experimental methods and simulations.* denotes membrane viscosity obtained from the bulk viscosity, $\eta$, using the relation $\eta_{m}=\eta h$, where $h$ is the bilayer thickness. For DMPC, SOPC and DOPC, the bilayer thickness are 3.67 nm, $4.00 \mathrm{~nm}$ [77] and $3.67 \mathrm{~nm}$ [62] respectively. $L_{d}$ and $L_{o}$ refer to liquid-disordered and liquid-ordered phases respectively. GUVs, LUVs and SUVs refer to Giant-Unilamellar vesicles, Large-Unilamellar vesicles and Small-Unilamellar vesicles respectively. Zero-frequency membrane viscosities obtained from the electrodeformation method developed in this study were measured at $E_{0}=8 \mathrm{kV} / \mathrm{m}\left(\gamma=\dot{5} 0 s^{-1}\right)$.

\begin{tabular}{|c|c|c|c|c|}
\hline Method & Membrane Composition & Viscosity (nPa.s.m) & Temperature & System \\
\hline Falling Ball viscosimetry 78 & SOPC & 3 & $298 \mathrm{~K}$ & GUVs \\
\hline Coarse-grained simulations 27 & SOPC & 3 & $323 \mathrm{~K}$ & Bilayer \\
\hline Electrodeformation (this study) & $\mathrm{SOPC}$ & $9.73 \pm 5.80$ & $298 \mathrm{~K}$ & GUVs \\
\hline Probe Diffusion [54] & SOPC & $13.2^{*}$ & $293 \mathrm{~K}$ & GUVs \\
\hline Optical Dynamometry 25 & DMPC & $5 \pm 2$ & $298 \mathrm{~K}$ & GUVs \\
\hline Neutron Spin Echo 19 & DMPC & 75 & $298 \mathrm{~K}$ & SUVs \\
\hline Coarse-grained simulations 27 & DMPC & 5 & $323 \mathrm{~K}$ & Bilayer \\
\hline Fluorescence Spectroscopy 79 & DMPC & $0.36^{*}$ & $298 \mathrm{~K}$ & SUVs \\
\hline Tether pulling 11 & $\mathrm{EPC}$ & $2.7-88$ & $295-298 \mathrm{~K}$ & GUVs \\
\hline Shear surface rheology 10 & POPC & 300000 & NA & Monolayer \\
\hline Electrodeformation (this study) & POPC & $9.32 \pm 5.95$ & $298 \mathrm{~K}$ & GUVs \\
\hline Shear surface rheology 10 & POPC:Chol $(7: 3)$ & 10000 & NA & Monolayer \\
\hline Shear surface rheology 10 & $\mathrm{DPPC}$ & 900000 & NA & Monolayer \\
\hline Optical Tweezers 80 & DOPC & $<0.6$ & $293 \mathrm{~K}$ & Bilayer \\
\hline Fluorescence Spectroscopy 79 & DOPC & $0.20^{*}$ & $298 \mathrm{~K}$ & SUVs \\
\hline Fluorescence Quantum Yield 81 & DOPC & $0.84^{*}$ & $298 \mathrm{~K}$ & LUVs \\
\hline Fluorescence lifetime of dye 82 & DOPC & $0.15^{*}$ & $298 \mathrm{~K}$ & LUVs \\
\hline All-atom simulations 7 & DOPC & $0.197 \pm 0.0069$ & $297 \mathrm{~K}$ & Bilayer \\
\hline Coarse-grained simulations 27] & DOPC & 0.17 & $323 \mathrm{~K}$ & Bilayer \\
\hline Membrane-anchored particles [16] & $\mathrm{DOPC}$ & $15.3 \pm 3.4$ & $297 \mathrm{~K}$ & GUVs \\
\hline Electrodeformation (this study) & DOPC & $4.11 \pm 2.63$ & $298 \mathrm{~K}$ & GUVs \\
\hline Probe Diffusion 15 & DOPC & $0.59 \pm 0.2$ & $298 \mathrm{~K}$ & GUVs \\
\hline Neutron Spin Echo 9 & DOPC & $16.7 \pm 1.1$ & $298 \mathrm{~K}$ & SUVs \\
\hline Electrodeformation (this study) & OMPC & $7.73 \pm 3.09$ & $298 \mathrm{~K}$ & GUVs \\
\hline Membrane-anchored particles [16] & 13:0 PC & $14.7 \pm 6.9$ & $297 \mathrm{~K}$ & GUVs \\
\hline Neutron Spin Echo 9 & DOPC:Chol (8:2) & $31.9 \pm 3.5$ & $298 \mathrm{~K}$ & SUVs \\
\hline Electrodeformation (this study) & DOPC:Chol $(1: 1)$ & $7.00 \pm 4.77$ & $298 \mathrm{~K}$ & GUVs \\
\hline Electrodeformation (this study) & DPPC:Chol $(1: 1)$ & $56.4 \pm 4.63$ & $298 \mathrm{~K}$ & GUVs \\
\hline Optical Tweezers 80 & DOPC:DPPC (2:1), $L_{o}$ & 2.1 & $293 \mathrm{~K}$ & Bilayer \\
\hline Shear Driven Flow 8 & DOPC:DPPC (85:15), $L_{d}$ & $1.9 \pm 11.9$ & $296 \mathrm{~K}$ & GUVs \\
\hline Shear Driven Flow 8 & DiPhyPC:Chol:DPPC $(5: 40: 55), L_{o}$ & $15.7 \pm 9.9$ & $296 \mathrm{~K}$ & GUVs \\
\hline Domain fluctuations 17 & DiPhyPC:Chol:DPPC (25:55:20) & $4 \pm 1$ & $293 \mathrm{~K}$ & GUVs \\
\hline Diffusion of domains 12 & DPPC:DOPC:Chol $(3.5: 3.5: 3), L_{o}$ & $10-500$ & $295 \mathrm{~K}$ & GUVs \\
\hline Diffusion of domains 13 & DiPhyPC:DPPC (1:1) & $2.2 \pm 0.1$ & $296.5 \mathrm{~K}$ & GUVs \\
\hline Shear Driven Flow 18 & DPPC:DOPC:Chol $(3: 6: 1), L_{o}$ domain & 4.1 & $293 \mathrm{~K}$ & GUVs \\
\hline Shear Driven Flow 18 & DPPC:DOPC:Chol $(4: 5: 1), L_{o}$ domain & 14 & $293 \mathrm{~K}$ & GUVs \\
\hline Shear Driven Flow 18 & DPPC:DOPC:Chol $(2: 6: 2), L_{o}$ domain & 9.6 & $293 \mathrm{~K}$ & GUVs \\
\hline Shear Driven Flow 18 & DPPC:DOPC:Chol $(4: 4: 2), L_{o}$ domain & 4.3 & $293 \mathrm{~K}$ & GUVs \\
\hline Shear Driven Flow 18 & DPPC:DOPC:Chol $(5: 3: 2), L_{o}$ domain & 54 & $293 \mathrm{~K}$ & GUVs \\
\hline Shear Driven Flow 18 & DPPC:DOPC:Chol $(6: 2: 2), L_{d}$ domain & 1200 & $293 \mathrm{~K}$ & GUVs \\
\hline Shear Driven Flow 18 & DPPC:DOPC:Chol $(2: 5: 3), L_{o}$ domain & 7.7 & $293 \mathrm{~K}$ & GUVs \\
\hline Shear Driven Flow 18 & DPPC:DOPC:Chol $(5: 2: 3), L_{d}$ domain & 85 & $293 \mathrm{~K}$ & GUVs \\
\hline Shear Driven Flow 18 & DPPC:DOPC:Chol $(6: 1: 3), L_{d}$ domain & 1200 & $293 \mathrm{~K}$ & GUVs \\
\hline Shear Driven Flow 18 & DPPC:DOPC:Chol (3:3:4), $L_{d}$ domain & 0.63 & $293 \mathrm{~K}$ & GUVs \\
\hline Shear Driven Flow [18] & DPPC:DOPC:Chol $(4: 2: 4), L_{d}$ domain & 65 & $293 \mathrm{~K}$ & GUVs \\
\hline Shear Driven Flow 18 & DPPC:DOPC:Chol $(5: 1: 4), L_{d}$ domain & 71 & $293 \mathrm{~K}$ & GUVs \\
\hline Shear Driven Flow 18 & DPPC:DOPC:Chol $(4: 3: 3), L_{d}$ domain & 98 & $293 \mathrm{~K}$ & GUVs \\
\hline Shear Driven Flow 18 & DPPC:DOPC:Chol $(3: 5: 2), L_{o}$ domain & 65 & $293 \mathrm{~K}$ & GUVs \\
\hline Electrodeformation (this study) & DPPC:DOPC:Chol $(1: 1: 1)$ & $17.7 \pm 3.06$ & $298 \mathrm{~K}$ & GUVs \\
\hline Electrodeformation (this study) & DPPC:DOPC:Chol (1:1:2) & $15.4 \pm 2.40$ & $298 \mathrm{~K}$ & GUVs \\
\hline Falling Ball viscosimetry 83 & $\mathrm{PBd}_{33}-b-\mathrm{PEO}_{20}$ & $1500 \pm 120$ & $298 \mathrm{~K}$ & GUVs \\
\hline Electrodeformation (this study) & $\mathrm{PBd}_{33}-b-\mathrm{PEO}_{20}$ & $2890 \pm 670$ & $298 \mathrm{~K}$ & GUVs \\
\hline Micropipette Aspiration 84 & PEG-b-PA6ester 1 & $7900000 \pm 200000$ & $298 \mathrm{~K}$ & GUVs \\
\hline Micropipette Aspiration 84 & PEG-b-PA444 & $4000000 \pm 200000$ & $298 \mathrm{~K}$ & GUVs \\
\hline Atomic Force Microscopy 85 & $\mathrm{PBd}_{130}-b-\mathrm{PEO}_{66}$ & 5000000 & $298 \mathrm{~K}$ & Bilayer \\
\hline Atomic Force Microscopy 85 & $\mathrm{PBd}_{130}-b-\mathrm{PEO}_{66}$ (cross linked) & 20000000 & $298 \mathrm{~K}$ & Bilayer \\
\hline Electrodeformation (this study) & $\mathrm{PBd}_{13}-b-\mathrm{PEO}_{11}$ & $14.4 \pm 4.40$ & $298 \mathrm{~K}$ & GUVs \\
\hline Electrodeformation (this study) & $\mathrm{PBd}_{22}-b-\mathrm{PEO}_{14}$ & $686 \pm 51.0$ & $298 \mathrm{~K}$ & GUVs \\
\hline Electrodeformation (this study) & $\mathrm{PBd}_{46}-b-\mathrm{PEO}_{24}$ & $20600 \pm 4700$ & $298 \mathrm{~K}$ & GUVs \\
\hline Electrodeformation (this study) & $\mathrm{PBd}_{54}-b-\mathrm{PEO}_{29}$ & $46700 \pm 900$ & $298 \mathrm{~K}$ & GUVs \\
\hline Electrodeformation (this study) & $\mathrm{PBd}_{120}-b-\mathrm{PEO}_{78}$ & $157000 \pm 54500$ & $298 \mathrm{~K}$ & GUVs \\
\hline
\end{tabular}


TABLE III. Membrane bending rigidity and capacitance of phospholipids, polymers $\mathrm{PBd}_{x}-b$ - $\mathrm{PEO}_{y}$ and mixed system of DOPC:DPPC:Chol at $25{ }^{\circ} \mathrm{C}$ determined in this study. Bending rigidity was measured with flickering spectroscopy and membrane capacitance was measured with the electrodeformation method. $M_{w}$ and $M_{h}$ refer to the total and hydrophobic molecular weight, respectively. NA means not available.

\begin{tabular}{|c|c|c|c|c|}
\hline Composition & $M_{w}[\mathrm{kDa}]$ & $M_{h}[\mathrm{kDa}]$ & $\kappa\left(k_{B} T\right)$ & $C_{m}\left(\mu F / \mathrm{cm}^{2}\right)$ \\
\hline \hline POPC & 0.760 & 0.448 & $27.8 \pm 2.3$ & $0.72 \pm 0.04$ \\
\hline SOPC & 0.787 & 0.476 & $30.1 \pm 3.1$ & $0.71 \pm 0.02$ \\
\hline DOPC & 0.786 & 0.474 & $22.2 \pm 2.0$ & $0.72 \pm 0.04$ \\
\hline OMPC & 0.732 & 0.420 & $27.1 \pm 2.6$ & $0.71 \pm 0.03$ \\
\hline DOPC:Chol $(1: 1)$ & NA & NA & $27.8 \pm 4.6$ & $0.50 \pm 0.09$ \\
\hline DPPC:Chol $(1: 1)$ & NA & NA & $121.3 \pm 11.0$ & $0.45 \pm 0.05$ \\
\hline DOPC:DPPC:Chol $(1: 1: 1)$ & NA & NA & $72.0 \pm 8.4$ & $0.51 \pm 0.16$ \\
\hline DOPC:DPPC:Chol $(1: 1: 2)$ NA & NA & $69.2 \pm 7.9$ & $0.63 \pm 0.10$ \\
\hline PBd $_{13}-b-\mathrm{PEO}_{11}$ & 1.19 & 0.7 & $17.1 \pm 1.5$ & $0.36 \pm 0.05$ \\
\hline PBd $_{22}-b-\mathrm{PEO}_{14}$ & 1.80 & 1.35 & $31.0 \pm 5.1$ & $0.27 \pm 0.03$ \\
\hline PBd $_{33}-b-\mathrm{PEO}_{20}$ & 2.60 & 1.85 & $54.4 \pm 6.4$ & $0.23 \pm 0.04$ \\
\hline PBd $_{46}-b-\mathrm{PEO}_{24}$ & 3.54 & 2.60 & NA & $0.18 \pm 0.03$ \\
\hline $\mathrm{PBd}_{54}-b-\mathrm{PEO}_{29}$ & 4.19 & 3.10 & $154 \pm 16.0$ & $0.18 \pm 0.04$ \\
\hline $\mathrm{PBd}_{120}-b-\mathrm{PEO}_{78}$ & 9.91 & 6.80 & NA & $0.07 \pm 0.01$ \\
\hline
\end{tabular}

TABLE IV. Membrane viscosities and values of a dye diffusion coefficient (DiC18) for the DOPC:DPPC:Chol ternary system. The values in brackets indicate lipid molar ratios (first column) and the number of measured vesicles (third column). All the experiments were performed at $25.0{ }^{\circ} \mathrm{C} . L_{d}$ and $L_{o}$ denote liquid disordered and liquid ordered, respectively. The diffusion coefficient were taken from 55

\begin{tabular}{|cccc|}
\hline Multi-component & Phase state & $\eta_{m}[\mathrm{nPa.s.m}]$ & $\mathrm{D}\left[\mu \mathrm{m}^{2} / \mathrm{s}[55]\right.$ \\
\hline \hline DOPC & $L_{d}$ & $4.11 \pm 2.63(20)$ & $6.30 \pm 0.13$ \\
DPPC:Chol (1:1) & $L_{o}$ & $56.4 \pm 4.63(25)$ & $0.48 \pm 0.06$ \\
DOPC:DPPC:Chol (1:1:2) & $L_{o}$ & $15.4 \pm 2.40(25)$ & $1.85 \pm 0.13$ \\
DOPC:DPPC:Chol (1:1:1) & $L_{d}$ & $17.7 \pm 3.06(18)$ & $2.50 \pm 0.20$ \\
DOPC:Chol (1:1) & $L_{d}$ & $7.00 \pm 4.77(25)$ & $3.25 \pm 0.25$ \\
\hline
\end{tabular}


A)

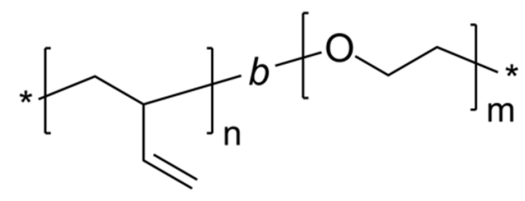

C)

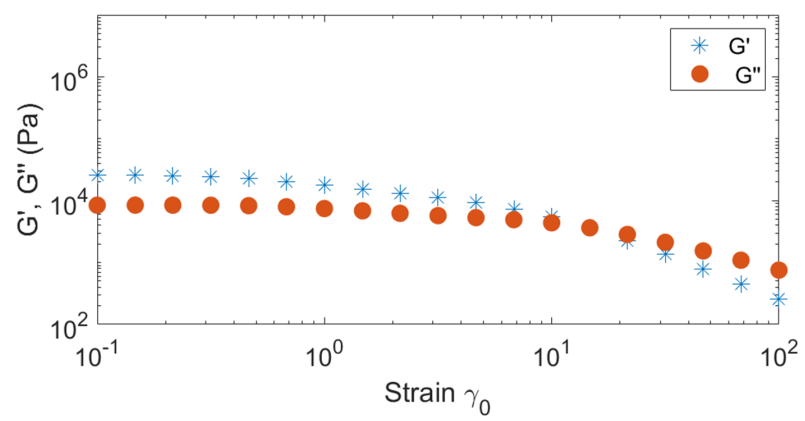

B)

D)
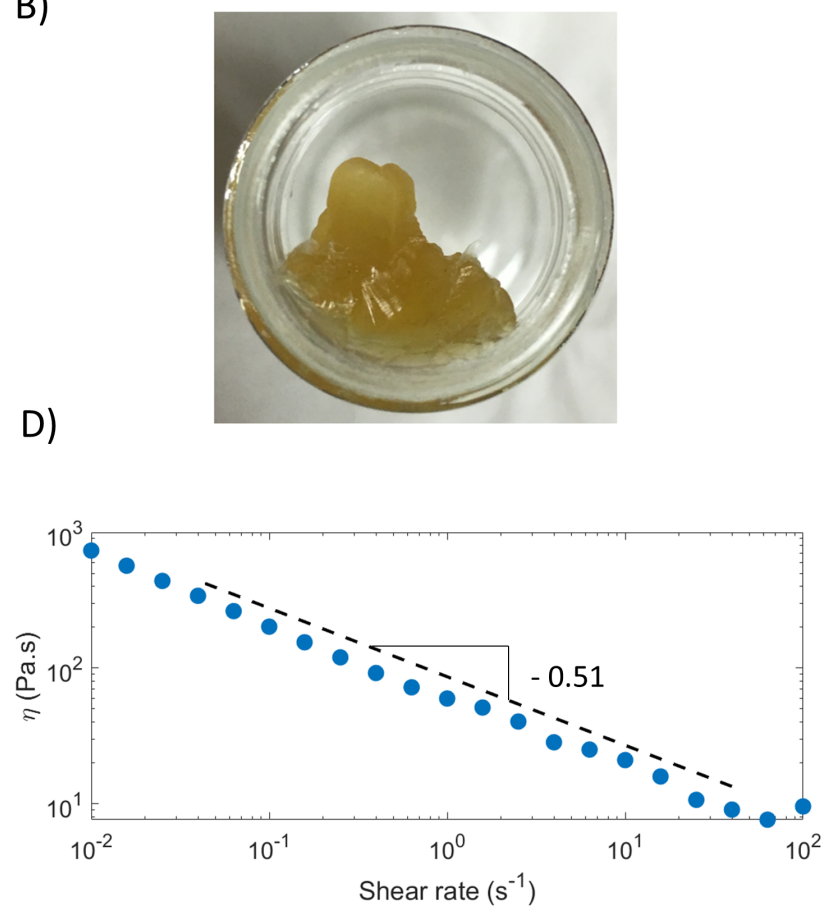

FIG. 11. A) Molecular structure of $\mathrm{PBd}_{n}-b-\mathrm{PEO}_{m}$ diblock co-polymer. B) The appearance of the polymer melt at room temperature. C) Determination of loss and storage modulus at different strain to determine the linear viscoelastic strain limit. D) The flow curve experiment with bulk viscosity vs shear rate. 
[1] S. J. Singer and Garth L. Nicolson. The fluid mosaic model of the structure of cell membranes. Science, 175(4023):720-731, 1972. ISSN 0036-8075. doi: 10.1126/science.175.4023.720.

[2] Adam E. Cohen and Zheng Shi. Do cell membranes flow like honey or jiggle like jello? BioEssays, 42(1):1900142, 2020.

[3] Anabel-Lise Le Roux, Xarxa Quiroga, Nikhil Walani, Marino Arroyo, and Pere Roca-Cusachs. The plasma membrane as a mechanochemical transducer. Philosophical Transactions of the Royal Society B: Biological Sciences, 374(1779): 20180221, 2019. doi:10.1098/rstb.2018.0221. URL https://royalsocietypublishing.org/doi/abs/10. 1098/rstb.2018.0221

[4] Rukshala Illukkumbura, Tom Bland, and Nathan W. Goehring. Patterning and polarization of cells by intracellular flows. Current Opinion in Cell Biology, 62:123 - 134, 2020. ISSN 0955-0674. doi: https://doi.org/10.1016/j.ceb.2019.10.005 URL http://www.sciencedirect.com/science/article/ pii/S0955067419300924

[5] Michael Sinensky. Homeoviscous adaptation-a homeostatic process that regulates the viscosity of membrane lipids in escherichia coli. Proceedings of the National Academy of Sciences, 71(2):522-525, 1974. ISSN 00278424. doi:10.1073/pnas.71.2.522. URL https://www. pnas.org/content/71/2/522

[6] J R Hazel. Thermal adaptation in biological membranes: Is homeoviscous adaptation the explanation? Annual Review of Physiology, 57(1):19-42, 1995. doi: 10.1146/annurev.ph.57.030195.000315. URL https: //doi.org/10.1146/annurev.ph.57.030195.000315 PMID: 7778864 .

[7] Andrew Zgorski, Richard W. Pastor, and Edward Lyman. Surface shear viscosity and interleaflet friction from nonequilibrium simulations of lipid bilayers. Journal of

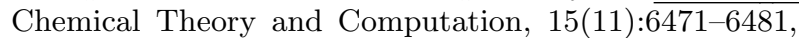
2019. doi:10.1021/acs.jctc.9b00683. URL https ://doi. org/10.1021/acs.jctc.9b00683. PMID: 31476126.

[8] Aurelia R. Honerkamp-Smith, Francis G. Woodhouse, Vasily Kantsler, and Raymond E. Goldstein. Membrane viscosity determined from shear-driven flow in giant vesicles. Phys. Rev. Lett., 111:038103, Jul 2013. doi: 10.1103/PhysRevLett.111.038103. URL https://link. aps.org/doi/10.1103/PhysRevLett.111.038103

[9] Saptarshi Chakraborty, Milka Doktorova, Trivikram R. Molugu, Frederick A. Heberle, Haden L. Scott, Boris Dzikovski, Michihiro Nagao, Laura-Roxana Stingaciu, Robert F. Standaert, Francisco N. Barrera, John Katsaras, George Khelashvili, Michael F. Brown, and Rana Ashkar. How cholesterol stiffens unsaturated lipid membranes. PNAS, 117(36):21896-21905, 2020. ISSN 00278424.

[10] Gabriel Espinosa, Iván López-Montero, Francisco Monroy, and Dominique Langevin. Shear rheology of lipid monolayers and insights on membrane fluidity. PNAS, 108(15):6008-6013, 2011. ISSN 0027-8424.

[11] R.E. Waugh. Surface viscosity measurements from large bilayer vesicle tether formation. ii. experiments. Biophysical Journal, 38(1):29 - 37, 1982. ISSN 0006-
3495. doi:https://doi.org/10.1016/S0006-3495(82)84527X URL http://www.sciencedirect.com/science/ article/pii/S000634958284527X

[12] Pietro Cicuta, Sarah L. Keller, and Sarah L. Veatch. Diffusion of liquid domains in lipid bilayer membranes. The Journal of Physical Chemistry B, 111(13):3328-3331, 2007.

[13] Eugene P. Petrov, Rafayel Petrosyan, and Petra Schwille. Translational and rotational diffusion of micrometersized solid domains in lipid membranes. Soft Matter, 8:7552-7555, 2012. doi:10.1039/C2SM25796C URL http://dx.doi.org/10.1039/C2SM25796C.

[14] Stephan Block. Brownian Motion at Lipid Membranes: A Comparison of Hydrodynamic Models Describing and Experiments Quantifying Diffusion within Lipid Bilayers. Biomolecules, 8(2), JUN 2018.

[15] C. Herold, P. Schwille, and E. P. Petrov. Dna condensation at freestanding cationic lipid bilayers. Phys. Rev. Lett., 104:148102, Apr 2010. doi: 10.1103/PhysRevLett.104.148102 URL https://link. aps.org/doi/10.1103/PhysRevLett.104.148102.

[16] Tristan T. Hormel, Sarah Q. Kurihara, M. Kathleen Brennan, Matthew C. Wozniak, and Raghuveer Parthasarathy. Measuring lipid membrane viscosity using rotational and translational probe diffusion. Phys. Rev. Lett., 112:188101, May 2014. doi: 10.1103/PhysRevLett.112.188101. URL https://link. aps.org/doi/10.1103/PhysRevLett.112.188101.

[17] Brian A. Camley, Cinzia Esposito, Tobias Baumgart, and Frank L.H. Brown. Lipid bilayer domain fluctuations as a probe of membrane viscosity. Biophysical Journal, 99(6):L44 - L46, 2010. ISSN 0006-3495. doi:https://doi.org/10.1016/j.bpj.2010.07.007 URL http://www.sciencedirect.com/science/article/ pii/S0006349510008519

[18] Yuka Sakuma, Toshihiro Kawakatsu, Takashi Taniguchi, and Masayuki Imai. Viscosity landscape of phase-separated lipid membrane estimated from fluid velocity field. Biophysical Journal, 118 (7):1576 - 1587, 2020. ISSN 0006-3495. doi: https://doi.org/10.1016/j.bpj.2020.01.009. URL http://www.sciencedirect.com/science/article/ pii/S0006349520300333

[19] Michihiro Nagao, Elizabeth G. Kelley, Rana Ashkar, Robert Bradbury, and Paul D. Butler. Probing elastic and viscous properties of phospholipid bilayers using neutron spin echo spectroscopy. The Journal of Physical Chemistry Letters, 8(19):4679-4684, 2017.

[20] Elizabeth G. Kelley, Paul D. Butler, Rana Ashkar, Robert Bradbury, and Michihiro Nagao. Scaling relationships for the elastic moduli and viscosity of mixed lipid membranes. Proceedings of the National Academy of Sciences, 117(38):23365-23373, 2020. ISSN 0027-8424. doi:10.1073/pnas.2008789117. URL https://www.pnas . org/content/117/38/23365

[21] Michihiro Nagao, Elizabeth G. Kelley, Antonio Faraone, Makina Saito, Yoshitaka Yoda, Masayuki Kurokuzu, Shinichi Takata, and Makoto Setovand Paul D. Butler. Relationship between viscosity and acyl tail dynamics in lipid bilayers. Phys. Rev. Lett., page accepted, 2021.

[22] Yilei Wu, Martin tefl, Agnieszka Olzyska, Martin Hof, 
Gokhan Yahioglu, Philip Yip, Duncan R. Casey, Oscar Ces, Jana Humpolkov, and Marina K. Kuimova. Molecular rheometry: direct determination of viscosity in lo and ld lipid phases via fluorescence lifetime imaging. Phys. Chem. Chem. Phys., 15:14986-14993, 2013. doi:10.1039/C3CP51953H URL http://dx.doi.org/ 10.1039/C3CP51953H

[23] Grzegorz Chwastek, Eugene P. Petrov, and James Peter Senz. A method for high-throughput measurements of viscosity in sub-micrometer-sized membrane systems. ChemBioChem, 21(6):836-844, 2020. doi:https://doi.org/10.1002/cbic.201900510 URL https://chemistry-europe.onlinelibrary.wiley. com/doi/abs/10.1002/cbic.201900510.

[24] K Velikov, C Dietrich, A Hadjiisky, K Danov, and B Pouligny. Motion of a massive microsphere bound to a spherical vesicle. Europhysics Letters (EPL), 40(4): 405-410, nov 1997. doi:10.1209/epl/i1997-00479-1 URL https://doi.org/10.1209/epl/i1997-00479-1.

[25] R. Dimova, B. Pouligny, and C. Dietrich. Pretransitional effects in dimyristoylphosphatidylcholine vesicle membranes: Optical dynamometry study. Biophysical Journal, 79(1):340-356, Jul 2000. ISSN 0006-3495. doi:10.1016/S0006-3495(00)76296-5 URL https ://doi. org/10.1016/S0006-3495(00)76296-5

[26] R. Dimova, C. Dietrich, A. Hadjiisky, K. Danov, and B. Pouligny. Falling ball viscosimetry of giant vesicle membranes: Finite-size effects. The European Physical Journal B - Condensed Matter and Complex Systems, 12(4):589-598, Dec 1999. ISSN 1434-6036. doi:10.1007/s100510051042. URL https://doi.org/10. $1007 / \mathrm{s} 100510051042$

[27] W.K. den Otter and S.A. Shkulipa. Intermonolayer friction and surface shear viscosity of lipid bilayer membranes. Biophysical Journal, 93 (2):423 - 433, 2007. ISSN 0006-3495. doi: https://doi.org/10.1529/biophysj.107.105395 URL http://www.sciencedirect.com/science/article/ pii/S0006349507712961

[28] Martin Vögele, Jürgen Köfinger, and Gerhard Hummer. Hydrodynamics of diffusion in lipid membrane simulations. Phys. Rev. Lett., 120:268104, Jun 2018. doi: 10.1103/PhysRevLett.120.268104 URL https://link. aps.org/doi/10.1103/PhysRevLett.120.268104

[29] Andrey A. Gurtovenko, Matti Javanainen, Fabio Lolicato, and Ilpo Vattulainen. The devil is in the details: What do we really track in single-particle tracking experiments of diffusion in biological membranes? The Journal of Physical Chemistry Letters, 10(5):1005-1011, 2019. doi:10.1021/acs.jpclett.9b00065. URL https:// doi.org/10.1021/acs.jpclett.9b00065

[30] Krassimir D. Danov, Rumiana Dimova, and Bernard Pouligny. Viscous drag of a solid sphere straddling a spherical or flat surface. Physics of Fluids, 12(11): 2711-2722, 2000. doi:10.1063/1.1289692 URL https : //aip.scitation.org/doi/abs/10.1063/1.1289692

[31] Ali Naji, Alex J. Levine, and P.A. Pincus. Corrections to the saffman-delbrck mobility for membrane bound proteins. Biophysical Journal, 93 (11):L49-L51, 2007. ISSN 0006-3495. doi: https://doi.org/10.1529/biophysj.107.119222 URL https://www.sciencedirect.com/science/article/ pii/S0006349507716235

[32] K. A. Riske and R. Dimova. Electro-deformation and poration of giant vesicles viewed with high temporal resolution. Biophys. J., 88:1143-1155, 2005.

[33] S. Aranda, K. A. Riske, R. Lipowsky, and R. Dimova. Morphological transitions of vesicles induced by ac electric fields. Biophys. J., 95:L19-L21, 2008.

[34] P. F. Salipante and P. M. Vlahovska. Vesicle deformation in dc electric pulses. Soft Matter, 10:3386 -3393, 2014.

[35] Miao Yu, Rafael B. Lira, Karin A. Riske, Rumiana Dimova, and Hao Lin. Ellipsoidal Relaxation of Deformed Vesicles. Phys. Rev. Lett., 115(12), SEP 18 2015. ISSN 0031-9007.

[36] J. Deschamps, V. Kantsler, E. Segre, and V. Steinberg. Dynamics of a vesicle in general flow. PNAS, 106:1144411447, 2009.

[37] Anish Shenoy, Christopher V. Rao, and Charles M. Schroeder. Stokes trap for multiplexed particle manipulation and assembly using fluidics. PNAS, 113(15):39763981, 2016. ISSN 0027-8424.

[38] Dinesh Kumar, Channing M. Richter, and Charles M. Schroeder. Conformational dynamics and phase behavior of lipid vesicles in a precisely controlled extensional flow. Soft Matter, 16:337-347, 2020. doi: 10.1039/C9SM02048A URL http://dx.doi.org/10. 1039/C9SM02048A

[39] Jochen Guck, Revathi Ananthakrishnan, Hamid Mahmood, Tess J. Moon, C. Casey Cunningham, and Josef Ks. The optical stretcher: A novel laser tool to micromanipulate cells. Biophysical Journal, 81(2):767-784, 2001. ISSN 0006-3495. doi:https://doi.org/10.1016/S00063495(01)75740-2. URL https://www.sciencedirect. com/science/article/pii/S0006349501757402

[40] Ulysse Delabre, Kasper Feld, Eleonore Crespo, Graeme Whyte, Cecile Sykes, Udo Seifert, and Jochen Guck. Deformation of phospholipid vesicles in an optical stretcher. Soft Matter, 11:6075-6088, 2015. doi: 10.1039/C5SM00562K URL http://dx.doi.org/10. 1039/C5SM00562K

[41] Mark L. Henle and Alex J. Levine. Effective viscosity of a dilute suspension of membrane-bound inclusions. Physics of Fluids, 21(3):033106, 2009. doi:10.1063/1.3086831. URL https://doi .org/10.1063/1.3086831

[42] Francis G. Woodhouse and Raymond E. Goldstein. Shear-driven circulation patterns in lipid membrane vesicles. Journal of Fluid Mechanics, 705:165175, 2012. doi: 10.1017/jfm.2012.118.

[43] Mohammad Rahimi, Antonio DeSimone, and Marino Arroyo. Curved fluid membranes behave laterally as effective viscoelastic media. Soft Matter, 9:11033-11045, 2013. doi:10.1039/C3SM51748A. URL http://dx.doi. org/10.1039/C3SM51748A

[44] Jon Karl Sigurdsson and Paul J. Atzberger. Hydrodynamic coupling of particle inclusions embedded in curved lipid bilayer membranes. Soft Matter, 12:6685-6707, 2016. doi:10.1039/C6SM00194G. URL http://dx.doi. org/10.1039/C6SM00194G.

[45] Petia M. Vlahovska and C. Misbah. Theory of vesicle dynamics in flow and electric fields. In R. Dimova and C. Marques, editors, The Giant Vesicle Book, page Chapter 7. CRC Press, 2019.

[46] P. M. Vlahovska. Non-equilibrium dynamics of lipid membranes: deformation and stability in electric fields. In A. Iglic, editor, Advances in Planar Lipid Bilayers and Liposomes, vol. 12, pages 103-146. Elsevier, 2010.

[47] P. M. Vlahovska. Dynamics of membrane bound parti- 
cles: capsules and vesicles. In C. Duprat and H.A. Stone, editors, Low-Reynolds-Number Flows: Fluid-Structure Interactions. 2016.

[48] D. Lacoste, G. I. Menon, M. Z. Bazant, and J. F. Joanny. Electrostatic and electrokinetic contributions to the elastic moduli of a driven membrane. Eur. Phys. J. E, 28: 243-264, 2009.

[49] C. Grosse and H. P. Schwan. Cellular membrane potentials induced by alternating fields. Biophys. J., 63 : 1632-1642, 1992.

[50] P. M. Vlahovska, R. S. Gracia, S. Aranda-Espinoza, and R. Dimova. Electrohydrodynamic model of vesicle deformation in alternating electric fields. Biophys. J., 96: 4789-4803, 2009.

[51] S. L. Veatch, S. L.and Keller. Organization in lipid membranes containing cholesterol. Phys.Rev.Lett., 89:268101, 2002.

[52] S. L. Veatch and S. L. Keller. Seeing spots: Complex phase behavior in simple membranes. BBA, 1746:172185, 2005.

[53] Pradeep Uppamoochikkal, Stephanie Tristram-Nagle, and John F. Nagle. Orientation of tie-lines in the phase diagram of dopc/dppc/cholesterol model biomembranes. Langmuir, 26(22):17363-17368, Nov 2010. ISSN 07437463. doi:10.1021/la103024f. URL https://doi.org/ 10.1021/la103024f

[54] Y. Gambin, R. Lopez-Esparza, M. Reffay, E. Sierecki, N. S. Gov, M. Genest, R. S. Hodges, and W. Urbach. Lateral mobility of proteins in liquid membranes revisited. PNAS, 103(7):2098-2102, 2006. ISSN 0027-8424. doi:10.1073/pnas.0511026103 URL https://www.pnas. org/content/103/7/2098

[55] Dag Scherfeld, Nicoletta Kahya, and Petra Schwille. Lipid dynamics and domain formation in model membranes composed of ternary mixtures of unsaturated and saturated phosphatidylcholines and cholesterol. Biophysical Journal, 85(6):3758 - 3768, 2003. ISSN 00063495. doi:https://doi.org/10.1016/S0006-3495(03)747912. URL http://www.sciencedirect.com/science/ article/pii/S0006349503747912.

[56] P G Saffman and M Delbrück. Brownian motion in biological membranes. PNAS, 72(8):3111-3113, 1975. ISSN 0027-8424. doi:10.1073/pnas.72.8.3111. URL https: //www.pnas.org/content/72/8/3111.

[57] Stephanie Tristram-Nagle, Horia I. Petrache, and John F. Nagle. Structure and interactions of fully hydrated dioleoylphosphatidylcholine bilayers. Biophysical Journal, $75(2): 917 \quad-925, \quad 1998 . \quad$ ISSN 00063495. doi:https://doi.org/10.1016/S0006-3495(98)775800. URL http://www.sciencedirect.com/science/ article/pii/S0006349598775800.

[58] John F. Nagle and Stephanie Tristram-Nagle. Structure of lipid bilayers. Biochimica et Biophysica Acta (BBA) - Reviews on Biomembranes, 1469(3):159 - 195, 2000. ISSN 0304-4157. doi:https://doi.org/10.1016/S03044157(00)00016-2 URL http://www.sciencedirect. com/science/article/pii/S0304415700000162.

[59] Yufeng Liu and John F. Nagle. Diffuse scattering provides material parameters and electron density profiles of biomembranes. Phys. Rev. E, 69:040901, Apr 2004. doi:10.1103/PhysRevE.69.040901. URL https://link. aps.org/doi/10.1103/PhysRevE.69.040901.

[60] Norbert Kucerka, Stephanie Tristram-Nagle, and John F. Nagle. Structure of fully hydrated fluid phase lipid bi- layers with monounsaturated chains. The Journal of Membrane Biology, 208(3):193-202, 2006. ISSN 14321424. doi:10.1007/s00232-005-7006-8 URL https:// doi.org/10.1007/s00232-005-7006-8

[61] Norbert Kučerka, John F. Nagle, Jonathan N. Sachs, Scott E. Feller, Jeremy Pencer, Andrew Jackson, and John Katsaras. Lipid bilayer structure determined by the simultaneous analysis of neutron and x-ray scattering data. Biophysical Journal, 95(5):2356-2367, Sep 2008. ISSN 0006-3495. URL http://www. sciencedirect.com/ science/article/pii/S0006349508783838.

[62] Jianjun Pan, Thalia T. Mills, Stephanie Tristram-Nagle, and John F. Nagle. Cholesterol perturbs lipid bilayers nonuniversally. Phys. Rev. Lett., 100:198103, May 2008. doi:10.1103/PhysRevLett.100.198103 URL https:// link.aps.org/doi/10.1103/PhysRevLett.100.198103

[63] Brian A. Camley and Frank L. H. Brown. Motion of objects embedded in lipid bilayer membranes: Advection and effective viscosity. The Journal of Chemical Physics, 151(12):124104, 2019.

[64] D. E. Discher and F. Ahmed. Polymersomes. Annu. Rev. Biomed. Eng., 8:323-341, 2006.

[65] Caterina LoPresti, Hannah Lomas, Marzia Massignani, Thomas Smart, and Giuseppe Battaglia. Polymersomes: nature inspired nanometer sized compartments. J. Mater. Chem., 19:3576-3590, 2009. doi:10.1039/B818869F. URL http://dx.doi.org/10.1039/B818869F.

[66] D. Boal. Mechanics of the Cell. Cambridge University Press, 2002.

[67] W. Rawicz, K.C. Olbrich, T. McIntosh, D. Needham, and E. Evans. Effect of chain length and unsaturation on elasticity of lipid bilayers. Biophys. J., 79:328-339, 2000.

[68] Harry Bermudez, Aaron K. Brannan, Daniel A. Hammer, Frank S. Bates, and Dennis E. Discher. Molecular weight dependence of polymersome membrane structure, elasticity, and stability. Macromolecules, 35(21): 8203-8208, 2002. doi:10.1021/ma0206691 URL https: //doi.org/10.1021/ma0206691

[69] B. M. Discher, Y. Y. Won, D. S. Ege, J. C. M. Lee, F. S. Bates, D. E. Discher, and D. A. Hammer. Polymersomes: Tough vesicles made from diblock copolymers. Science, 284:1143-1146, 1999.

[70] P. Fischer and P. Erni. Emulsion drops in external flow fields - the role of liquid interfaces. Curr. Opinion Coll. Interface Sci., 12:196-205, 2007.

[71] Robert Bradbury and Michihiro Nagao. Effect of charge on the mechanical properties of surfactant bilayers. Soft Matter, 12:9383-9390, 2016. doi:10.1039/C6SM01686C URL http://dx.doi.org/10.1039/C6SM01686C.

[72] Miglena I. Angelova and Dimiter S. Dimitrov. Liposome electroformation. Faraday Discuss. Chem. Soc., 81:303311, 1986. doi:10.1039/DC9868100303. URL http://dx. doi.org/10.1039/DC9868100303.

[73] Hammad A. Faizi, Rumiana Dimova, and Petia M. Vlahovska. Electromechanical characterization of biomimetic membranes using electrodeformation of vesicles. Electrophoresis, 0:1-6, 2021. doi:https://doi.org/10.1002/elps.202100091 URL https://doi.org/10.1002/elps.202100091

[74] Rubn Serral Gracia, Natalya Bezlyepkina, Roland L. Knorr, Reinhard Lipowsky, and Rumiana Dimova. Effect of cholesterol on the rigidity of saturated and unsaturated membranes: fluctuation and electrodeformation analysis 
of giant vesicles. Soft Matter, 6:1472-1482, 2010. doi: 10.1039/B920629A URL http://dx.doi.org/10.1039/ B920629A

[75] Hammad A. Faizi, Shelli L. Frey, Jan Steinkhler, Rumiana Dimova, and Petia M. Vlahovska. Bending rigidity of charged lipid bilayer membranes. Soft Matter, 15(29):6006-6013, 2019. ISSN 1744-683X. doi: 10.1039/C9SM00772E URL http://dx.doi.org/10. 1039/C9SM00772E

[76] Hammad A. Faizi, Cody J. Reeves, Vasil N. Georgiev, Petia M. Vlahovska, and Rumiana Dimova. Fluctuation spectroscopy of giant unilamellar vesicles using confocal and phase contrast microscopy. Soft Matter, 16:89969001, 2020. doi:10.1039/D0SM00943A URL http:// dx.doi.org/10.1039/D0SM00943A.

[77] Norbert Kuerka, Mu-Ping Nieh, and John Katsaras. Fluid phase lipid areas and bilayer thicknesses of commonly used phosphatidylcholines as a function of temperature. Biochimica et Biophysica Acta (BBA) - Biomembranes, 1808 (11):2761-2771, 2011. ISSN 0005-2736. doi: https://doi.org/10.1016/j.bbamem.2011.07.022 URL https://www.sciencedirect.com/science/article/ pii/S0005273611002276

[78] R Dimova, C Dietrich, A Hadjiisky, K Danov, and B Pouligny. Falling ball viscosimetry of giant vesicle membranes: Finite-size effects. EUROPEAN PHYSICAL JOURNAL B, 12(4):589-598, DEC 1999. ISSN 1434-6028. doi:10.1007/s100510051042.

[79] Yuki Nojima and Koichi Iwata. Viscosity heterogeneity inside lipid bilayers of single-component phosphatidylcholine liposomes observed with picosecond time-resolved fluorescence spectroscopy. The Journal of Physical Chemistry B, 118(29):8631-8641, 2014. doi: 10.1021/jp503921e URL https://doi.org/10.1021/ jp503921e. PMID: 24967901.

[80] Guillermo J. Amador, Dennis van Dijk, Roland Kieffer, Marie-Eve Aubin-Tam, and Daniel Tam. Hydrodynamic shear dissipation and transmission in lipid bilayers. Proceedings of the National Academy of Sciences, 118(21), 2021. ISSN 0027-8424. doi: 10.1073/pnas.2100156118. URL https://www.pnas . org/content/118/21/e2100156118

[81] Yilei Wu, Martin Stefl, Agnieszka Olzynska, Martin Hof,
Gokhan Yahioglu, Philip Yip, Duncan R. Casey, Oscar Ces, Jana Humpolícková, and Marina K. Kuimova. Molecular rheometry: direct determination of viscosity in lo and ld lipid phases via fluorescence lifetime imaging. Physical Chemistry Chemical Physics, 15(36):1498614993, 2013. ISSN 1463-9076. doi:10.1039/C3CP51953H URL http: //dx.doi .org/10.1039/C3CP51953H.

[82] Grzegorz Chwastek, Eugene P. Petrov, and James Peter Senz. A method for high-throughput measurements of viscosity in sub-micrometer-sized membrane systems. ChemBioChem, 21(6):836-844, 2020. doi: 10.1002/cbic.201900510. URL https : //onlinelibrary . wiley.com/doi/abs/10.1002/cbic.201900510

[83] R. Dimova, U. Seifert, B. Pouligny, S. Förster, and H.G. Döbereiner. Hyperviscous diblock copolymer vesicles. The European Physical Journal E, 7(3):241-250, Mar 2002. ISSN 1292-8941. doi:10.1140/epje/i200101032 URL https ://doi.org/10.1140/epje/i200101032.

[84] Elyes Mabrouk, Damien Cuvelier, Lea-Laetitia Pontani, Bing Xu, Daniel Lvy, Patrick Keller, Franoise BrochardWyart, Pierre Nassoy, and Min-Hui Li. Formation and material properties of giant liquid crystal polymersomes. Soft Matter, 5:1870-1878, 2009. doi:10.1039/B815817G URL http://dx.doi.org/10.1039/B815817G.

[85] Marta Kocun, Waltraut Mueller, Michael Maskos, Ingo Mey, Burkhard Geil, Claudia Steinem, and Andreas Janshoff. Viscoelasticity of pore-spanning polymer membranes derived from giant polymersomes. Soft Matter, 6:2508-2516, 2010. doi:10.1039/B924650A. URL http: //dx.doi.org/10.1039/B924650A

[86] Petia M. Vlahovska. Electrohydrodynamics of drops and vesicles. Annu. Rev. Fluid Mech., 51:305-330, 2019.

[87] P. F. Salipante, R. Knorr, R. Dimova, and P. M. Vlahovska. Electrodeformation method for measuring the capacitance of bilayer membranes. Soft Matter, 8:3810 3816, 2012.

[88] Matthew A. Wade, Dylan Walsh, Johnny Ching-Wei Lee, Elizabeth Kelley, Katie Weigandt, Damien Guironnet, and Simon A. Rogers. Color, structure, and rheology of a diblock bottlebrush copolymer solution. Soft Matter, 16: 4919-4931, 2020. doi:10.1039/D0SM00397B URL http: //dx.doi.org/10.1039/D0SM00397B 